\title{
A Coupled Legendre-Laguerre Spectral-Element Method for the Navier-Stokes Equations in Unbounded Domains
}

\author{
Qingqu Zhuang • Jie Shen · Chuanju Xu
}

Received: 7 November 2008 / Revised: 17 June 2009 / Accepted: 29 June 2009 /

Published online: 9 July 2009

(C) Springer Science+Business Media, LLC 2009

\begin{abstract}
A coupled Legendre-Laguerre spectral element method is proposed for the Stokes and Navier-Stokes equations in unbounded domains. The method combines advantages of the high accuracy of the Laguerre-spectral method for unbounded domains and the geometric flexibility of the spectral-element method. Rigorous stability and error analysis for the Stokes problem is carried out. Numerical results indicate that the proposed method is very effective for some realistic flow problems in unbounded domains, such as flows passing a circular cylinder.
\end{abstract}

Keywords Laguerre functions · Legendre polynomials · Navier-Stokes equations · Stokes equations · Inf-sup condition · Error analysis · Spectral element · Computational fluid dynamics

\section{Introduction}

There have been many theoretical and numerical investigations on using spectral methods for solving PDEs in unbounded domains. For an overview on this subject, we refer to the book by Boyd [3] and a more recent review paper by Shen and Wang [18]. However, to the best of the authors' knowledge, essentially no effort has been made on using a spectral method for direct simulation of flow problems in unbounded domains. A first step in this direction was made in a recent paper [1] where the authors studied the Legendre-Laguerre

The work of J. Shen was partially supported by NFS grant DMS-0610646 and AFOSR grant FA9550-08-1-0416.

The research of C. Xu was partially supported by National NSF of China under Grant 10531080, the Excellent Young Teachers Program by the Ministry of Education of China, and 973 High Performance Scientific Computation Research Program 2005CB321703.

Q. Zhuang · C. Xu ( $₫)$

School of Mathematical Sciences, Xiamen University, 361005 Xiamen, China

e-mail: cjxu@xmu.edu.cn

J. Shen

Department of Mathematics, Purdue University, West Lafayette, IN 47907, USA 
spectral method for the Stokes equations in a semi-infinite channel. In that paper, the authors constructed stable discretization spaces using Legendre and Laguerre series for the Stokes problem and derived a sharp estimate for the inf-sup constant. However, due to the onedomain approach, the results in [1] can not be directly used for complex geometries such as those encountered in simulating flows passing obstacles.

In this paper we continue the work started in [1] and aim at developing a coupled Legendre-Laguerre spectral-element method for Stokes and Navier-Stokes equations in complex unbounded domains. The method will combine advantages of the high accuracy of the Laguerre-spectral method for unbounded domains and the geometric flexibility of the spectral-element method. In order to validate the method and show that it can be applied to realistic flow problems, we will implement it to simulate flows passing a cylinder and compare the results with the approach using an artificial boundary with non-reflecting boundary conditions [20].

On the theoretical side, we shall carry out stability and error analysis of the coupled Legendre-Laguerre spectral-element method for the Stokes problems in a simple geometry, for the sake of the clarity. It is evident that this analysis can be extended to complex geometries by using standard arguments of the spectral-element method.

The outline of this paper is as follows. In Sect. 2 we present the Stokes problem in a semiinfinite channel and formulate the coupled Legendre-Laguerre spectral-element method for the Stokes problem. In Sect. 3, we study the stability of the coupled Legendre-Laguerre spectral-element method and provide an explicit lower bound for the inf-sup constant. Then, we carry out a rigorous error analysis for the proposed method in Sect. 4. We provide in Sect. 5 implementation details of the method for the Stokes and time-dependent NavierStokes equations, and present several sets of numerical results, including numerical simulation for the flow passing a circular cylinder.

We now introduce some notations/conventions which will be used throughout the paper. Let $\Omega$ be an open connected domain, and $\omega>0$ be a weight function on $\Omega$, and we denote by $(u, v)_{\Omega, \omega}:=\int_{\Omega} u v \omega d \Omega$ the inner product of $L_{\omega}^{2}(\Omega)$ whose norm is denoted by $\|\cdot\|_{\omega, \Omega}$. We set $L_{0, \omega}^{2}(\Omega)=\left\{v \in L_{\omega}^{2}(\Omega): \int_{\Omega} v \omega d \Omega=0\right\}$. We use $H_{\omega}^{m}(\Omega)$ and $H_{0, \omega}^{m}(\Omega)$ to denote the usual weighted Sobolev spaces with norm $\|\cdot\|_{m, \omega, \Omega}$. In cases where no confusion would arise, $\omega$ (if $\omega \equiv 1$ ) and $\Omega$ may be dropped from the notations. We denote by $c$ or $c_{j}$ generic positive constants independent of the discretization parameters. We also use the expression $A \lesssim B$ to mean that $A \leq c B$. Throughout this paper we will use boldface letters to denote vectors and vector functions.

\section{Stokes Problem in a Semi-Infinite Channel}

An important motivation for developing coupled Legendre-Laguerre spectral element method is to solve challenging problems in multiple-dimensional infinite domains. A particular application is to simulate the flows passing an obstacle in a semi-infinite channel. An essential step in developing numerical schemes for such problems is to study the Stokes problem in a semi-infinite channel.

To fix the idea, we consider the semi-infinite channel $\Omega=\{x: 0<x<+\infty,-1<y<$ 1 \} with boundary $\partial \Omega$. We define two subsets of $\Omega$ :

$$
\Omega^{-}=\{x: 0<x<a,-1<y<1\}, \quad \Omega^{+}=\{x: a<x<\infty,-1<y<1\} .
$$

We also denote $\Gamma^{k}=\partial \Omega \cap \partial \Omega^{k}(k= \pm)$ and $\Gamma_{0}=\partial \Omega^{-} \cap \partial \Omega^{+}$. 


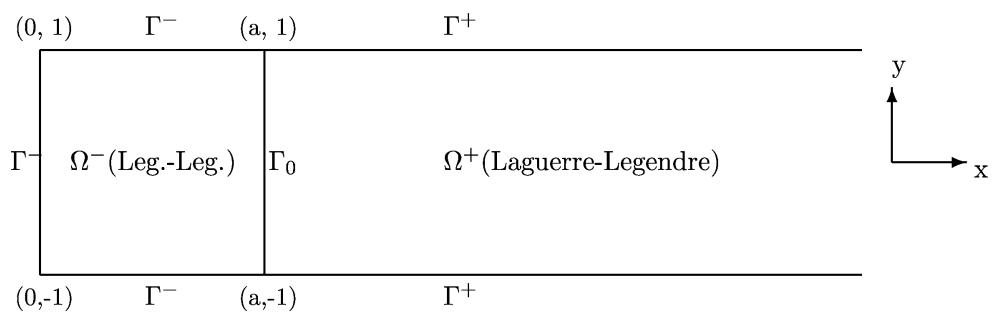

Fig. 1 Domain configuration for the coupled Legendre-Laguerre method

An illustration of the domain configuration is shown in Fig. 1.

For any function $v$ defined in $\Omega$, we use $v^{-}$and $v^{+}$to denote the restrictions of $v$ to $\Omega^{-}$ and $\Omega^{+}$, respectively. We define $(\cdot, \cdot)_{k}(k= \pm)$ and $(\cdot, \cdot)_{\Gamma}$ by

$$
\left(u^{k}, v^{k}\right)_{k}=\int_{\Omega^{k}} u^{k} v^{k} d \boldsymbol{x}, \quad(\varphi, \phi)_{\Gamma}=\int_{\Gamma} \varphi \phi d \sigma .
$$

Obviously, we have

$$
(u, v)_{\Omega}=\left(u^{-}, v^{-}\right)_{-}+\left(u^{+}, v^{+}\right)_{+} .
$$

Consider now the Stokes problem in the semi-infinite channel:

$$
\begin{cases}-\Delta \boldsymbol{u}+\nabla p=\boldsymbol{f} & \text { in } \Omega, \\ \nabla \cdot \boldsymbol{u}=0 & \text { in } \Omega, \\ \boldsymbol{u}_{\mid \partial \Omega}=0, & \\ \lim _{x \rightarrow \infty} \boldsymbol{u}=0 . & \end{cases}
$$

Its weak formulation is: find $(\boldsymbol{u}, p) \in H_{0}^{1}(\Omega)^{2} \times L^{2}(\Omega)$, such that

$$
\begin{cases}(\nabla \boldsymbol{u}, \nabla \boldsymbol{v})-(\nabla \cdot \boldsymbol{v}, p)=(\boldsymbol{f}, \boldsymbol{v}) & \forall \boldsymbol{v} \in H_{0}^{1}(\Omega)^{2}, \\ (\nabla \cdot \boldsymbol{u}, q)=0 & \forall q \in L^{2}(\Omega) .\end{cases}
$$

Now we define the coupled Legendre-Laguerre approximation. Let $\mathcal{L}_{i}(x)$ and $L_{j}(y)$ be respectively the Laguerre and Legendre polynomial of degree $i$ and $j$, and $\hat{\mathcal{L}}_{i}(x)=$ $\mathcal{L}_{i}(x) \exp (-x / 2)$. We recall the following orthogonality relation:

$$
\int_{-1}^{1} \int_{a}^{\infty} \hat{\mathcal{L}}_{i}(x-a) L_{j}(y) \hat{\mathcal{L}}_{m}(x-a) L_{n}(y) d x d y=\frac{2}{2 n+1} \delta_{i m} \delta_{j n} .
$$

Let $\mathbb{P}_{N}\left(\Omega^{-}\right)$be the space of all polynomials of degree $\leq N$ in $\Omega^{-}$with respect to each variable $x$ and $y$. We define the space $\hat{\mathbb{P}}_{M, N}\left(\Omega^{+}\right)$by

$$
\hat{\mathbb{P}}_{M, N}\left(\Omega^{+}\right):=\operatorname{span}\left\{\hat{\mathcal{L}}_{i}(x-a) L_{j}(y), i=0,1, \ldots, M ; j=0,1, \ldots, N\right\} .
$$

Let us denote by $\mathcal{N}$ the pair of parameters $(M, N)$, and define

$$
\begin{aligned}
& X_{\mathcal{N}}=H_{0}^{1}(\Omega)^{2} \cap\left\{\boldsymbol{v}: \boldsymbol{v}^{-} \in \mathbb{P}_{N}\left(\Omega^{-}\right)^{2}, \boldsymbol{v}^{+} \in \hat{\mathbb{P}}_{M, N}\left(\Omega^{+}\right)^{2}\right\}, \\
& M_{\mathcal{N}}=\left\{q: q^{-} \in \mathbb{P}_{N-2}\left(\Omega^{-}\right), q^{+} \in \hat{\mathbb{P}}_{M-1, N-2}\left(\Omega^{+}\right)\right\} .
\end{aligned}
$$


We define the discrete inner product $(\cdot, \cdot)_{\mathcal{N}}$ by

$$
\begin{aligned}
(\phi, \psi)_{\mathcal{N}} & =\left(\phi^{-}, \psi^{-}\right)_{\mathcal{N},-}+\left(\phi^{+}, \psi^{+}\right)_{\mathcal{N},+} \\
& =\sum_{m=0}^{N} \sum_{n=0}^{N} \phi^{-}\left(\xi_{m n}^{-}\right) \psi^{-}\left(\xi_{m n}^{-}\right) \omega_{m n}^{-}+\sum_{p=0}^{M} \sum_{q=0}^{N} \phi^{+}\left(\xi_{p q}^{+}\right) \psi^{+}\left(\xi_{p q}^{+}\right) \omega_{p q}^{+},
\end{aligned}
$$

where

$$
\begin{array}{ll}
\xi_{m n}^{-}=\left(\xi_{1, m}^{-}, \xi_{2, n}^{-}\right), & \omega_{m n}^{-}=\left(\omega_{1, m}^{-}, \omega_{2, n}^{-}\right), \quad 0 \leq m, n \leq N, \\
\xi_{p q}^{+}=\left(\hat{\xi}_{1, p}^{+}, \xi_{2, q}^{+}\right), & \omega_{p q}^{+}=\left(\hat{\omega}_{1, p}^{+}, \omega_{2, q}^{+}\right), \quad 0 \leq p \leq M, 0 \leq q \leq N,
\end{array}
$$

with $\xi_{1, m}^{-}$and $\omega_{1, m}^{-}$(resp. $\xi_{2, n}^{-}$and $\omega_{2, n}^{-}$or $\xi_{2, q}^{+}$and $\omega_{2, q}^{+}$) the Legendre-Gauss-Lobatto points and the associated weights in $[0, a]$ (resp. $[-1,1])$. While $\hat{\xi}_{1, p}^{+}$and $\hat{\omega}_{1, p}^{+}$the Laguerre-GaussRadau points and the associated weights in $[a,+\infty)$. The associated discrete norms are denoted by $\|\cdot\|_{\mathcal{N}},\|\cdot\|_{\mathcal{N},-}$ and $\|\cdot\|_{\mathcal{N},+}$.

Then, the coupled Legendre-Laguerre approximation to (2.2) is: Find $\boldsymbol{u}_{\mathcal{N}} \in X_{\mathcal{N}}, p_{\mathcal{N}} \in$ $M_{\mathcal{N}}$, such that

$$
\begin{cases}\left(\nabla \boldsymbol{u}_{\mathcal{N}}, \nabla \boldsymbol{v}_{\mathcal{N}}\right)_{\mathcal{N}}-\left(\nabla \cdot \boldsymbol{v}_{\mathcal{N}}, p_{\mathcal{N}}\right)_{\mathcal{N}}=\left(\boldsymbol{f}, \boldsymbol{v}_{\mathcal{N}}\right)_{\mathcal{N}} & \forall \boldsymbol{v}_{\mathcal{N}} \in X_{\mathcal{N}} \\ \left(\nabla \cdot \boldsymbol{u}_{\mathcal{N}}, q_{\mathcal{N}}\right)_{\mathcal{N}}=0 & \forall q_{\mathcal{N}} \in M_{\mathcal{N}}\end{cases}
$$

The coercivity and continuity of the bilinear form $\left(\nabla \boldsymbol{w}_{\mathcal{N}}, \nabla \boldsymbol{v}_{\mathcal{N}}\right)_{\mathcal{N}}$ and the continuity of the bilinear form $\left(\nabla \cdot v_{\mathcal{N}}, q_{\mathcal{N}}\right)_{\mathcal{N}}$ can be verified easily by using the property of the discrete norm associated to the Legendre-Gauss-Lobatto quadrature [2] and the fact that

$$
\int_{0}^{\infty} p q d x=\sum_{k=0}^{M} p\left(\hat{\xi}_{k}\right) q\left(\hat{\xi}_{k}\right) \hat{\omega}_{k}, \quad \forall p, q \in \hat{\mathbb{P}}_{M}\left(R^{+}\right),
$$

where $\left\{\hat{\xi}_{k}, \hat{\omega}_{k}\right\}$ are the Laguerre-Radau points and weights in $[0, \infty)$, and

$$
\hat{\mathbb{P}}_{M}\left(R^{+}\right)=\operatorname{span}\left\{\hat{\mathcal{L}}_{0}(x), \hat{\mathcal{L}}_{1}(x), \ldots, \hat{\mathcal{L}}_{M}(x)\right\} .
$$

More precisely, we have

$$
\begin{aligned}
\left(\nabla \boldsymbol{v}_{\mathcal{N}}, \nabla \boldsymbol{v}_{\mathcal{N}}\right)_{\mathcal{N}} & =\left(\nabla \boldsymbol{v}_{\mathcal{N}}^{-}, \nabla \boldsymbol{v}_{\mathcal{N}}^{-}\right)_{\mathcal{N},-}+\left(\nabla \boldsymbol{v}_{\mathcal{N}}^{+}, \nabla \boldsymbol{v}_{\mathcal{N}}^{+}\right)_{\mathcal{N},+} \\
& \gtrsim\left\|\boldsymbol{v}_{\mathcal{N}}^{-}\right\|_{1,-}^{2}+\left\|\boldsymbol{v}_{\mathcal{N}}^{+}\right\|_{1,+}^{2}=\left\|\boldsymbol{v}_{\mathcal{N}}\right\|_{1, \Omega}^{2}, \quad \forall \boldsymbol{v}_{\mathcal{N}} \in X_{\mathcal{N}}, \\
\left(\nabla \boldsymbol{w}_{\mathcal{N}}, \nabla \boldsymbol{v}_{\mathcal{N}}\right)_{\mathcal{N}} & =\left(\nabla \boldsymbol{w}_{\mathcal{N}}^{-}, \nabla \boldsymbol{v}_{\mathcal{N}}^{-}\right)_{\mathcal{N},-}+\left(\nabla \boldsymbol{w}_{\mathcal{N}}^{+}, \nabla \boldsymbol{v}_{\mathcal{N}}^{+}\right)_{\mathcal{N},+} \\
& \leq\left\|\nabla \boldsymbol{w}_{\mathcal{N}}^{-}\right\|_{\mathcal{N},-}\left\|\nabla \boldsymbol{v}_{\mathcal{N}}^{-}\right\|_{\mathcal{N},-}+\left\|\nabla \boldsymbol{w}_{\mathcal{N}}^{+}\right\|_{\mathcal{N},+}\left\|\nabla \boldsymbol{v}_{\mathcal{N}}^{+}\right\|_{\mathcal{N}_{,+}} \\
& \leq\left\|\nabla \boldsymbol{w}_{\mathcal{N}}\right\|_{\mathcal{N}}\left\|\nabla \boldsymbol{v}_{\mathcal{N}}\right\|_{\mathcal{N}} \lesssim\left\|\boldsymbol{w}_{\mathcal{N}}\right\|_{1, \Omega}\left\|\boldsymbol{v}_{\mathcal{N}}\right\|_{1, \Omega}, \quad \forall \boldsymbol{w}_{\mathcal{N}}, \boldsymbol{v}_{\mathcal{N}} \in X_{\mathcal{N}}, \\
\left(\nabla \cdot \boldsymbol{v}_{\mathcal{N}}, q_{\mathcal{N}}\right)_{\mathcal{N}} & =\left(\nabla \cdot \boldsymbol{v}_{\mathcal{N}}^{-}, q_{\mathcal{N}}^{-}\right)_{\mathcal{N},-}+\left(\nabla \cdot \boldsymbol{v}_{\mathcal{N}}^{+}, q_{\mathcal{N}}^{+}\right)_{\mathcal{N},+} \\
& \lesssim\left\|\nabla \cdot \boldsymbol{v}_{\mathcal{N}}^{-}\right\|_{\mathcal{N},-}\left\|q_{\mathcal{N}}^{-}\right\|_{\mathcal{N},-}+\left\|\nabla \cdot \boldsymbol{v}_{\mathcal{N}}^{+}\right\|_{\mathcal{N},+}\left\|q_{\mathcal{N}}^{+}\right\|_{\mathcal{N},+} \\
& \lesssim\left\|\boldsymbol{v}_{\mathcal{N}}\right\|_{1, \Omega}\left\|q_{\mathcal{N}}\right\|_{0, \Omega}, \quad \forall \boldsymbol{v}_{\mathcal{N}} \in X_{\mathcal{N}}, q_{\mathcal{N}} \in M_{\mathcal{N}}
\end{aligned}
$$


Then it is well-known that the well-posedness of the coupled formulation (2.4) relies on the so called inf-sup condition [4]:

$$
\inf _{q_{\mathcal{N}} \in M_{\mathcal{N}}} \sup _{\boldsymbol{v}_{\mathcal{N}} \in X_{\mathcal{N}}} \frac{-\left(\nabla \cdot \boldsymbol{v}_{\mathcal{N}}, q_{\mathcal{N}}\right)_{\mathcal{N}}}{\left\|\boldsymbol{v}_{\mathcal{N}}\right\|_{1, \Omega}\left\|q_{\mathcal{N}}\right\|_{0, \Omega}} \geq \beta_{\mathcal{N}}>0,
$$

where $\beta_{\mathcal{N}}$ is the inf-sup constant.

\section{Estimation of the inf-sup Constant}

This section is devoted to the estimation of the inf-sup constant.

\section{Theorem 3.1}

$$
\inf _{q \in M_{\mathcal{N}}} \sup _{\boldsymbol{v} \in X_{\mathcal{N}}} \frac{-(\nabla \cdot \boldsymbol{v}, q)_{\mathcal{N}}}{\|\boldsymbol{v}\|_{1, \Omega}\|q\|_{0, \Omega}} \gtrsim \min \left(N^{-\frac{1}{2}}, M^{-1}\right)
$$

Proof Let us first remark that

$$
(\nabla \cdot v, q)_{\mathcal{N}}=(\nabla \cdot v, q), \quad \forall q \in M_{\mathcal{N}}, v \in X_{\mathcal{N}}
$$

Consequently, it suffices to prove $(3.1)$ with $(\nabla \cdot v, q)$ in place of $(\nabla \cdot v, q)_{\mathcal{N}}$.

Let $q \in M_{\mathcal{N}}$. We start with $\Omega^{-}$and decompose $q^{-}$as

$$
q^{-}=q_{0}^{-}+r^{-}
$$

such that $q_{0}^{-} \in L_{0}^{2}\left(\Omega^{-}\right) \cap \mathbb{P}_{N-2}\left(\Omega^{-}\right)$and $r^{-}$is a constant. Then, it is known (cf. [2]) that there exist a positive constant $\beta_{N}^{-} \sim N^{-\frac{1}{2}}$ and a function $v_{0}^{-} \in H_{0}^{1}\left(\Omega^{-}\right)^{2} \cap \mathbb{P}_{N}\left(\Omega^{-}\right)^{2}$, such that

$$
\nabla \cdot v_{0}^{-}=-q_{0}^{-} \quad \text { and } \quad\left\|v_{0}^{-}\right\|_{1, \Omega^{-}} \lesssim \frac{1}{\beta_{N}^{-}}\left\|q_{0}^{-}\right\|_{0, \Omega^{-}} .
$$

Given a function $g \in X_{\mathcal{N}}$ satisfying

$$
\int_{\Gamma_{0}} \boldsymbol{g} \cdot \boldsymbol{n}^{-} d \sigma=\left|\Omega^{-}\right|
$$

where $\left|\Omega^{-}\right|$is the measure of $\Omega^{-}$. We denote by $\boldsymbol{w}_{0}^{-}$a function in $H_{0}^{1}\left(\Omega^{-}\right)^{2} \cap \mathbb{P}_{N}\left(\Omega^{-}\right)^{2}$ such that

$$
\left(\nabla \cdot \boldsymbol{w}_{0}^{-}, q\right)_{-}=(\nabla \cdot \boldsymbol{g}, q)_{-}, \quad \forall q \in L_{0}^{2}\left(\Omega^{-}\right) \cap \mathbb{P}_{N-2}\left(\Omega^{-}\right) .
$$

Then define $\tilde{\boldsymbol{v}}^{-}:=\boldsymbol{g}-\boldsymbol{w}_{0}^{-}$, which satisfies

$$
\begin{aligned}
& \left(\nabla \cdot \tilde{\boldsymbol{v}}^{-}, q\right)_{-}=0, \quad \forall q \in L_{0}^{2}\left(\Omega^{-}\right) \cap \mathbb{P}_{N-2}\left(\Omega^{-}\right), \quad \text { and } \\
& \int_{\Gamma} \tilde{\boldsymbol{v}}^{-} \cdot \boldsymbol{n}^{-} d \sigma=\int_{\Gamma_{0}} \boldsymbol{g} \cdot \boldsymbol{n}^{-} d \sigma=\left|\Omega^{-}\right| .
\end{aligned}
$$

Denoting $\boldsymbol{v}^{-}=\boldsymbol{v}_{0}^{-}-r^{-} \tilde{\boldsymbol{v}}^{-}$, using (3.2), (3.3) and (3.6), we find that $\boldsymbol{v}^{-} \in H^{1}\left(\Omega^{-}\right)^{2} \cap$ $\mathbb{P}_{N}\left(\Omega^{-}\right)^{2}, \boldsymbol{v}^{-}{ }_{\mid \Gamma^{-}}=0, \boldsymbol{v}^{-}{ }_{\mid \Gamma_{0}}=r^{-} \boldsymbol{g}_{\mid \Gamma_{0}}$, and

$$
-\left(\nabla \cdot \boldsymbol{v}^{-}, q^{-}\right)_{-}=-\left(\nabla \cdot\left(\boldsymbol{v}_{0}^{-}-r^{-} \tilde{\boldsymbol{v}}^{-}\right), q_{0}^{-}+r^{-}\right)_{-}=\left(q_{0}^{-}, q_{0}^{-}\right)_{-}+\left(r^{-}\right)^{2} \int_{\Omega^{-}} \nabla \cdot \tilde{\boldsymbol{v}}^{-} d x d y .
$$


So we obtain

$$
-\left(\nabla \cdot v^{-}, q^{-}\right)_{-}=\left\|q_{0}^{-}\right\|_{0, \Omega^{-}}^{2}+\left(r^{-}\right)^{2}\left|\Omega^{-}\right| .
$$

We now consider $\Omega^{+}$. We recall from [1] that there exists a positive constant $\beta_{M}^{+} \sim M^{-1}$ and a function $\boldsymbol{v}_{0}^{+} \in H_{0}^{1}\left(\Omega^{+}\right)^{2} \cap \hat{\mathbb{P}}_{M, N}\left(\Omega^{+}\right)^{2}$ such that

$$
\nabla \cdot \boldsymbol{v}_{0}^{+}=-q^{+} \quad \text { and } \quad\left\|v_{0}^{+}\right\|_{1, \Omega^{+}} \lesssim \frac{1}{\beta_{M}^{+}}\left\|q^{+}\right\|_{0, \Omega^{+}} .
$$

For the same function $\boldsymbol{g} \in X_{\mathcal{N}}$ given in (3.4), let $\boldsymbol{w}_{0}^{+}$be a function in $H_{0}^{1}\left(\Omega^{+}\right)^{2} \cap \hat{\mathbb{P}}_{M, N}\left(\Omega^{+}\right)^{2}$ such that

$$
\left(\nabla \cdot \boldsymbol{w}_{0}^{+}, q\right)_{+}=(\nabla \cdot \boldsymbol{g}, q)_{+}, \quad \forall q \in \hat{\mathbb{P}}_{M-1, N-2}\left(\Omega^{+}\right) .
$$

Then define $\tilde{\boldsymbol{v}}^{+}:=\boldsymbol{g}-\boldsymbol{w}_{0}^{+}$which satisfies

$$
\tilde{\boldsymbol{v}}_{\mid \Gamma_{0}}^{+}=\boldsymbol{g}_{\mid \Gamma_{0}}, \quad \text { and } \quad\left(\nabla \cdot \tilde{\boldsymbol{v}}^{+}, q\right)_{+}=0, \quad \forall q \in \hat{\mathbb{P}}_{M-1, N-2}\left(\Omega^{+}\right) .
$$

Denoting $\boldsymbol{v}^{+}=\boldsymbol{v}_{0}^{+}-r^{-} \tilde{\boldsymbol{v}}^{+}$, using (3.8) and (3.10), we have $\boldsymbol{v}^{+} \in H^{1}\left(\Omega^{+}\right)^{2} \cap \hat{\mathbb{P}}_{M, N}\left(\Omega^{+}\right)^{2}$, $\boldsymbol{v}^{+}{ }_{\mid \Gamma^{+}}=0, \boldsymbol{v}^{+}{ }_{\mid \Gamma_{0}}=r^{-} \boldsymbol{g}_{\mid \Gamma_{0}}$, and

$$
-\left(\nabla \cdot \boldsymbol{v}^{+}, q^{+}\right)_{+}=-\left(\nabla \cdot\left(\boldsymbol{v}_{0}^{+}-r^{-} \tilde{\boldsymbol{v}}^{+}\right), q^{+}\right)_{+}=\left\|q^{+}\right\|_{0, \Omega^{+}}^{2} .
$$

Now define

$$
\boldsymbol{v}= \begin{cases}\boldsymbol{v}^{-} & \text {in } \Omega^{-}, \\ \boldsymbol{v}^{+} & \text {in } \Omega^{+}\end{cases}
$$

then $v \in X_{\mathcal{N}}$, and from (3.7) and (3.11), we find

$$
\begin{aligned}
-(\nabla \cdot \boldsymbol{v}, q) & =-\left(\nabla \cdot \boldsymbol{v}^{-}, q^{-}\right)_{-}-\left(\nabla \cdot \boldsymbol{v}^{+}, q^{+}\right)_{+} \\
& =\left\|q_{0}^{-}\right\|_{0, \Omega^{-}}^{2}+\left(r^{-}\right)^{2}\left|\Omega^{-}\right|+\left\|q^{+}\right\|_{0, \Omega^{+}}^{2} \\
& =\left\|q^{-}\right\|_{0, \Omega^{-}}^{2}+\left\|q^{+}\right\|_{0, \Omega^{+}}^{2}=\|q\|_{0, \Omega}^{2} .
\end{aligned}
$$

By the definitions of $\boldsymbol{v}^{-}$and $\boldsymbol{v}^{+}$, and using (3.3), (3.5), (3.8) and (3.9), we get

$$
\left\|\boldsymbol{v}^{-}\right\|_{1, \Omega^{-}}=\left\|\boldsymbol{v}_{0}^{-}-r^{-} \tilde{\boldsymbol{v}}^{-}\right\|_{1, \Omega^{-}} \lesssim \frac{1}{\beta_{N}^{-}}\left\|q_{0}^{-}\right\|_{0, \Omega^{-}}+\left|r^{-}\right|\left\|\tilde{\boldsymbol{v}}^{-}\right\|_{1, \Omega^{-}} \leq \frac{c_{1}}{\beta_{N}^{-}}\left\|q^{-}\right\|_{0, \Omega^{-}},
$$

and

$$
\left\|\boldsymbol{v}^{+}\right\|_{1, \Omega^{+}}=\left\|\boldsymbol{v}_{0}^{+}-r^{-} \tilde{\boldsymbol{v}}^{+}\right\|_{1, \Omega^{+}} \lesssim \frac{1}{\beta_{M}^{+}}\left\|q^{+}\right\|_{0, \Omega^{+}}+\left|r^{-}\right|\left\|\tilde{\boldsymbol{v}}^{+}\right\|_{1, \Omega^{+}} \leq c_{2}\left(\frac{1}{\beta_{M}^{+}}+\frac{1}{\beta_{N}^{-}}\right)\|q\|_{0, \Omega},
$$

where $c_{1}, c_{2}$ depend on $\boldsymbol{g}$ and $\left|\Omega^{-}\right|$. Combining the last three results leads to

$$
\frac{-(\nabla \cdot v, q)_{\mathcal{N}}}{\|v\|_{1, \Omega}} \geq \frac{\|q\|_{0, \Omega}^{2}}{\frac{c_{1}}{\beta_{N}^{-}}\left\|q^{-}\right\|_{0, \Omega^{-}}+c_{2}\left(\frac{1}{\beta_{M}^{+}}+\frac{1}{\beta_{N}^{-}}\right)\|q\|_{0, \Omega}} \geq \beta_{\mathcal{N}}\|q\|_{0, \Omega}
$$

where $\beta_{\mathcal{N}}=\min \left(\beta_{N}^{-}, \beta_{M}^{+}\right)=\min \left(N^{-\frac{1}{2}}, M^{-1}\right)$. 


\section{Error Estimates}

We carry out in this section error analysis for the coupled Legendre-Laguerre spectral element method to the Stokes problem.

\subsection{Some Preliminary Approximation Results}

We start with some notations and definitions which are needed in the following error analysis. Let $\omega_{r}(x)=x^{r} e^{-x}$ and $\hat{\omega}_{r}(x)=x^{r}$. We now recall some approximation results collected in the recent review paper [18] for the Laguerre functions $\left\{\hat{\mathcal{L}}_{k}\right\}$, which correspond to the generalized Laguerre functions $\left\{\hat{\mathcal{L}}_{k}^{(\alpha)}\right\}$ in [18] with index $\alpha=0$.

We first introduce some suitable functional spaces. Let us denote $\hat{\partial}_{x}=\partial_{x}+\frac{1}{2}$. We define for all $m \geq 1$,

$$
\hat{B}^{m}\left(R^{+}\right):=\left\{v: \hat{\partial}_{x}^{k} v \in L_{\hat{\omega}_{k}}^{2}\left(R^{+}\right), 0 \leq k \leq m\right\},
$$

equipped with the following norm and semi-norm

$$
\|v\|_{\hat{B}^{m}}=\left(\sum_{k=0}^{m}\left\|\hat{\partial}_{x}^{k} v\right\|_{\hat{\omega}_{k}}^{2}\right)^{1 / 2}, \quad|v|_{\hat{B}^{m}}=\left\|\hat{\partial}_{x}^{m} v\right\|_{\hat{\omega}_{m}} .
$$

We also define

$$
\hat{B}_{*}^{m}\left(R^{+}\right):=\left\{v: \hat{\partial}_{x} v \in \hat{B}^{m-1}\left(R^{+}\right)\right\},
$$

endowed with the norm and semi-norm:

$$
\|v\|_{\hat{B}_{*}^{m}}=\left\|\hat{\partial}_{x} v\right\|_{\hat{B}^{m-1}}, \quad|v|_{\hat{B}_{*}^{m}}=\left|\hat{\partial}_{x} v\right|_{\hat{B}^{m-1}} .
$$

Let $\pi_{M, x}$ be the $L_{\omega_{0}}^{2}$-orthogonal projector from $L_{\omega_{0}}^{2}\left(R^{+}\right)$into $\mathbb{P}_{M}\left(R^{+}\right)$defined by:

$$
\int_{0}^{\infty}\left(v-\pi_{M, x} v\right) \phi_{M} \omega_{0} d x=0, \quad \forall v \in L_{\omega_{0}}^{2}\left(R^{+}\right), \phi_{M} \in \mathbb{P}_{M}\left(R^{+}\right) .
$$

Then, we define the operator $\hat{\pi}_{M, x}$ from $L^{2}\left(R^{+}\right)$into $\hat{\mathbb{P}}_{M}\left(R^{+}\right)$by (cf. [17]):

$$
\hat{\pi}_{M, x} v(x)=e^{-x / 2} \pi_{M, x}\left(v(x) e^{x / 2}\right), \quad \forall v \in L^{2}\left(R^{+}\right) .
$$

It can be easily verified that

$$
\begin{aligned}
& \int_{0}^{\infty}\left(v-\hat{\pi}_{M, x} v\right) \phi_{M} d x=\int_{0}^{\infty}\left(v(x) e^{x / 2}-\pi_{M, x}\left(v(x) e^{x / 2}\right)\right) e^{-x / 2} \phi_{M} d x=0, \\
& \quad \forall \phi_{M} \in \hat{\mathbb{P}}_{M}\left(R^{+}\right) .
\end{aligned}
$$

Consequently, $\hat{\pi}_{M, x}$ is the orthogonal projector from $L^{2}\left(R^{+}\right)$into $\hat{\mathbb{P}}_{M}\left(R^{+}\right)$. The following result is a special case $(\alpha=0)$ of Theorem 3.3 in [18]:

Lemma 4.1 For all $v \in \hat{B}^{m}\left(R^{+}\right), m \geq 0$, we have

$$
\left\|\hat{\partial}_{x}^{l}\left(v-\hat{\pi}_{M, x} v\right)\right\|_{\hat{\omega}_{l}} \lesssim M^{(l-m) / 2}\left\|\hat{\partial}_{x}^{m} v\right\|_{\hat{\omega}_{m}}, \quad 0 \leq l \leq m .
$$


Next, we set $W_{M}=\left\{v \in \mathbb{P}_{M}\left(R^{+}\right): v(0)=0\right\}$ and define $\pi_{M, x}^{1,0}: H_{0, \omega_{0}}^{1}\left(R^{+}\right) \rightarrow W_{M}$ by:

$$
\int_{0}^{\infty}\left(v-\pi_{M, x}^{1,0} v\right)^{\prime} \phi_{M}^{\prime} \omega_{0} d x=0 \quad \forall \phi_{M} \in W_{M} .
$$

Then, we set $\hat{W}_{M}=\left\{v e^{-x / 2}: v \in W_{M}\right\}$ and define $\hat{\pi}_{M, x}^{1,0}: H_{0}^{1}\left(R^{+}\right) \rightarrow \hat{W}_{M}$ by

$$
\hat{\pi}_{M, x}^{1,0} v(x):=e^{-x / 2} \pi_{M, x}^{1,0}\left(v(x) e^{x / 2}\right), \quad \forall v \in H_{0}^{1}\left(R^{+}\right) .
$$

We can verify that

$$
\left(\left(v-\hat{\pi}_{M, x}^{1,0} v\right)^{\prime}, v_{M}^{\prime}\right)+\frac{1}{4}\left(v-\hat{\pi}_{M, x}^{1,0} v, v_{M}\right)=0, \quad \forall v \in H_{0}^{1}\left(R^{+}\right), v_{M} \in \hat{W}_{M},
$$

and the following result holds (cf. Theorem 3.4 in [18]):

Lemma 4.2 For all $v \in H_{0}^{1}\left(R^{+}\right) \cap \hat{B}_{*}^{m}\left(R^{+}\right), m \geq 1$, we have

$$
\left\|v-\hat{\pi}_{M, x}^{1,0} v\right\|_{1} \lesssim M^{\frac{1}{2}-\frac{m}{2}}\left\|\hat{\partial}_{x}^{m} v\right\|_{\hat{\omega}_{m-1}} .
$$

Now let $\hat{I}_{M, x}: C\left(\bar{R}^{+}\right) \rightarrow \hat{\mathbb{P}}_{M}\left(R^{+}\right)$be the interpolation operator at the Laguerre GaussRadau points. The following result is a special case $(\alpha=0)$ of Theorem 3.8 in [18] (which was first proved in [11]):

Lemma 4.3 For all $v \in C\left(\bar{R}^{+}\right) \cap \hat{B}^{m}\left(R^{+}\right) \cap \hat{B}_{*}^{m}\left(R^{+}\right), m \geq 1$, we have

$$
\left\|v-\hat{I}_{M, x} v\right\|_{0} \lesssim M^{\frac{1}{2}-\frac{m}{2}}\left(\left\|\hat{\partial}_{x}^{m} v\right\|_{\hat{\omega}_{m-1}}+(\ln M)^{\frac{1}{2}}\left\|\hat{\partial}_{x}^{m} v\right\|_{\hat{\omega}_{m}}\right) .
$$

For any $v \in H^{1}\left(R^{+}\right)$, we set $\hat{v}(x)=v(x)-v(0) e^{-x / 2}$, and define the quasi-orthogonal projection operator $\hat{\pi}_{M, x}^{1}: H^{1}\left(R^{+}\right) \rightarrow \hat{\mathbb{P}}_{M}\left(R^{+}\right)$by

$$
\hat{\pi}_{M, x}^{1} v:=\hat{\pi}_{M, x}^{1,0} \hat{v}+v(0) e^{-x / 2}, \quad \forall v \in H^{1}\left(R^{+}\right) .
$$

Then we have the following result.

Lemma 4.4 For all $v \in H^{1}\left(R^{+}\right) \cap \hat{B}_{*}^{m}\left(R^{+}\right), m \geq 1$, we have

$$
\left\|v-\hat{\pi}_{M, x}^{1} v\right\|_{1} \lesssim M^{\frac{1}{2}-\frac{m}{2}}\left\|\hat{\partial}_{x}^{m} v\right\|_{\hat{\omega}_{m-1}} .
$$

Proof By definition, we have $\left(\hat{\pi}_{M, x}^{1} v\right)(0)=v(0)$, and $v-\hat{\pi}_{M, x}^{1} v=\hat{v}-\hat{\pi}_{M, x}^{1,0} \hat{v}$. We then derive immediately from Lemma 4.2 that

$$
\left\|v-\hat{\pi}_{M, x}^{1} v\right\|_{1}=\left\|\hat{v}-\hat{\pi}_{M, x}^{1,0} \hat{v}\right\|_{1} \lesssim M^{\frac{1}{2}-\frac{m}{2}}\left\|\hat{\partial}_{x}^{m} \hat{v}\right\|_{\hat{\omega}_{m-1}} .
$$

Furthermore, a direct calculation shows that

$$
\hat{\partial}_{x}^{m}\left(v(0) e^{-x / 2}\right)=v(0) \hat{\partial}_{x}^{m}\left(e^{-x / 2}\right)=0, \quad m \geq 1 .
$$


Thus

$$
\hat{\partial}_{x}^{m} \hat{v}=\hat{\partial}_{x}^{m} v-\hat{\partial}_{x}^{m}\left(v(0) e^{-x / 2}\right)=\hat{\partial}_{x}^{m} v, \quad m \geq 1 .
$$

This completes the proof.

We now recall some approximation results by Legendre polynomials. Let us denote $\Lambda=$ $(-1,1)$. Let $\Pi_{N}: L^{2}(\Lambda) \rightarrow \mathbb{P}_{N}(\Lambda)$ be the $L^{2}(\Lambda)$-orthogonal projector defined by

$$
\int_{-1}^{1}\left(v-\Pi_{N} v\right) \phi d z=0, \quad \forall \phi \in \mathbb{P}_{N}(\Lambda),
$$

and $\Pi_{N}^{1,0}: H_{0}^{1}(\Lambda) \rightarrow \mathbb{P}_{N}^{0}(\Lambda)$ be the $H_{0}^{1}(\Lambda)$-orthogonal projector defined by

$$
\int_{-1}^{1} \partial_{z}\left(v-\Pi_{N}^{1,0} v\right) \partial_{z} \phi d z=0, \quad \forall \phi \in \mathbb{P}_{N}^{0}(\Lambda) .
$$

We use $z$ here to denote either $x$ or $y$.

Also let $I_{N}: C(\bar{\Lambda}) \rightarrow \mathbb{P}_{N}(\Lambda)$ be the interpolation operator based on the Legendre-GaussLobatto points. Then, we have the following results (cf. $[8,10])$ :

\section{Lemma 4.5}

$$
\begin{aligned}
\left\|v-\Pi_{N} v\right\|_{0} & \lesssim N^{-s}\|v\|_{s}, \quad s \geq 0, v \in H^{s}(\Lambda) \\
\left\|v-\Pi_{N}^{1,0} v\right\|_{k} & \lesssim N^{k-s}\|v\|_{s}, \quad k=0,1, s \geq 1, v \in H_{0}^{1}(\Lambda) \cap H^{s}(\Lambda) \\
\left\|v-I_{N} v\right\|_{0} & \lesssim N^{-s}\|v\|_{s}, \quad s \geq 1, v \in C(\bar{\Lambda}), v \in H^{s}(\Lambda) .
\end{aligned}
$$

For any $v \in H^{1}(\Lambda)$, we set $\tilde{v}(z)=v(z)-v(-1) \frac{1-z}{2}-v(1) \frac{1+z}{2} \in H_{0}^{1}(\Lambda)$. We define a quasi-orthogonal projector $\Pi_{N}^{1}: H^{1}(\Lambda) \rightarrow P_{N}(\Lambda)$ by

$$
\Pi_{N}^{1} v:=\Pi_{N}^{1,0} \tilde{v}+v(-1) \frac{1-z}{2}+v(1) \frac{1+z}{2} .
$$

By definition, we have $\left(\Pi_{N}^{1} v\right)( \pm 1)=v( \pm 1)$, and $v-\Pi_{N}^{1} v=\tilde{v}-\Pi_{N}^{1,0} \tilde{v}$. We then derive immediately from Lemma 4.5 the following:

\section{Lemma 4.6}

$$
\left|v-\Pi_{N}^{1} v\right|_{1}+N\left\|v-\Pi_{N}^{1} v\right\|_{0} \lesssim N^{1-s}\|v\|_{s}, \quad s \geq 1, v \in H^{s}(\Lambda) .
$$

\subsection{Error Analysis}

Let $R_{a}^{+}=(a,+\infty)$. We denote by $L^{2}\left(\Lambda, \hat{B}^{m}\left(R_{a}^{+}\right)\right)$the space of the measurable functions $v: \Lambda \rightarrow \hat{B}^{m}\left(R_{a}^{+}\right)$such that

$$
\|v\|_{\hat{B}_{a}^{m} ; 0, \Omega^{+}}:=\left\{\int_{\Lambda}\|v(\cdot, y)\|_{\hat{B}^{m}, R_{a}^{+}}^{2} d y\right\}^{\frac{1}{2}}<\infty .
$$

In particular, the norm of $L^{2}\left(\Lambda, L^{2}\left(R_{a}^{+}\right)\right)$is denoted by $\|\cdot\|_{0, \Omega^{+}}$, which is defined by

$$
\|v\|_{0, \Omega^{+}}:=\|v\|_{0 ; 0, \Omega^{+}}:=\left(\int_{\Omega^{+}} v^{2} d \boldsymbol{x}\right)^{1 / 2} .
$$


Moreover, for any non-negative integer $n$, we define

$$
H^{n}\left(\Lambda, L^{2}\left(R_{a}^{+}\right)\right):=\left\{v: \frac{\partial^{j} v}{\partial y^{j}} \in L_{y}^{2}\left(\Lambda, L^{2}\left(R_{a}^{+}\right)\right), 0 \leq j \leq n\right\},
$$

the norm of this space is given by

$$
\|v\|_{0 ; n, \Omega^{+}}:=\left\{\sum_{j=0}^{n}\left\|\frac{\partial^{j} v}{\partial y^{j}}\right\|_{0, \Omega^{+}}^{2}\right\}^{\frac{1}{2}} .
$$

Finally for any non-negative integer $m, n$, we define the following spaces

$$
\begin{aligned}
& Y^{m ; n}\left(\Omega^{+}\right):=H^{n}\left(\Lambda, L^{2}\left(R_{a}^{+}\right)\right) \cap H^{n-1}\left(\Lambda, \hat{B}_{*}^{1}\left(R_{a}^{+}\right)\right) \cap H^{1}\left(\Lambda, \hat{B}_{*}^{m}\left(R_{a}^{+}\right)\right), \\
& A^{m ; n}\left(\Omega^{+}\right):=H^{n}\left(\Lambda, L^{2}\left(R_{a}^{+}\right)\right) \cap L^{2}\left(\Lambda, \hat{B}^{m}\left(R_{a}^{+}\right)\right), \\
& \bar{B}^{m ; n}\left(\Omega^{+}\right):=H^{n}\left(\Lambda, L^{2}\left(R_{a}^{+}\right)\right) \cap H^{1}\left(\Lambda, \hat{B}^{m}\left(R_{a}^{+}\right)\right) \cap H^{1}\left(\Lambda, \hat{B}_{*}^{m}\left(R_{a}^{+}\right)\right),
\end{aligned}
$$

endowed respectively with the following norms

$$
\begin{aligned}
& \|v\|_{Y_{+}^{m ; n}}=\left(\|v\|_{0 ; n, \Omega^{+}}^{2}+\|v\|_{\hat{B}_{*}^{1} ; n-1, \Omega^{+}}^{2}+\|v\|_{\hat{B}_{*}^{m} ; 1, \Omega^{+}}^{2}\right)^{\frac{1}{2}}, \\
& \|v\|_{A_{+}^{m ; n}}=\left\{\|v\|_{\hat{B}^{m} ; 0, \Omega^{+}}^{2}+\|v\|_{0 ; n, \Omega^{+}}^{2}\right\}^{\frac{1}{2}}, \\
& \|v\|_{\bar{B}_{+}^{m ; n}}=\left(\|v\|_{0 ; n, \Omega^{+}}^{2}+\|v\|_{\hat{B}^{m} ; 1, \Omega^{+}}^{2}+\|v\|_{\hat{B}_{*}^{m} ; 1, \Omega^{+}}^{2}\right)^{\frac{1}{2}} .
\end{aligned}
$$

We are now in a position to derive the error estimates.

Theorem 4.1 If the solution (u,p) of problem (2.2) satisfies $\boldsymbol{u} \in H_{0}^{1}(\Omega)^{2} \cap\left\{\boldsymbol{v}: \boldsymbol{v}^{-} \in\right.$ $\left.H^{\sigma}\left(\Omega^{-}\right)^{2}, \boldsymbol{v}^{+} \in Y^{m ; n}\left(\Omega^{+}\right)^{2}\right\}, \quad p \in\left\{p: p^{-} \in H^{\sigma-1}\left(\Omega^{-}\right), p^{+} \in A^{m-1 ; n-1}\left(\Omega^{+}\right)\right\}, \sigma \geq 1$, $m \geq 1, n \geq 1 ;$ And $\boldsymbol{f} \in\left\{\boldsymbol{v}: \boldsymbol{v}^{-} \in H^{\rho}\left(\Omega^{-}\right)^{2}, \boldsymbol{v}^{+} \in \bar{B}^{r ; s}\left(\Omega^{+}\right)^{2}\right\} \cap C(\Omega), \rho \geq 1, r \geq 1, s \geq 1$. Then the solution $\left(\boldsymbol{u}_{\mathcal{N}}, p_{\mathcal{N}}\right)$ of (2.4) admits the following error estimates:

$$
\begin{aligned}
\left\|\boldsymbol{u}-\boldsymbol{u}_{\mathcal{N}}\right\|_{1, \Omega} \lesssim & N^{1-\sigma}\left(\left\|\boldsymbol{u}^{-}\right\|_{\sigma, \Omega^{-}}+\left\|p^{-}\right\|_{\sigma-1, \Omega^{-}}\right)+N^{-\rho}\left\|\boldsymbol{f}^{-}\right\|_{\rho, \Omega^{-}} \\
& +\left(M^{\frac{1}{2}-\frac{m}{2}}+N^{1-n}\right)\left(M\left\|\boldsymbol{u}^{+}\right\|_{Y_{+}^{m ; n}}+\left\|p^{+}\right\|_{A_{+}^{m-1 ; n-1}}\right) \\
& +\left(\left((\ln M)^{\frac{1}{2}}+1\right) M^{\frac{1}{2}-\frac{r}{2}}+N^{-s}\right)\left\|\boldsymbol{f}^{+}\right\|_{\bar{B}_{+}^{r ; s}}, \\
\left\|p-p_{\mathcal{N}}\right\|_{0, \Omega} \lesssim & \frac{1}{\beta_{\mathcal{N}}}\left[N^{1-\sigma}\left(\left\|\boldsymbol{u}^{-}\right\|_{\sigma, \Omega^{-}}+\left\|p^{-}\right\|_{\sigma-1, \Omega^{-}}\right)+N^{-\rho}\left\|\boldsymbol{f}^{-}\right\|_{\rho, \Omega^{-}}\right. \\
& +\left(M^{\frac{1}{2}-\frac{m}{2}}+N^{1-n}\right)\left(M\left\|\boldsymbol{u}^{+}\right\|_{Y_{+}^{m ; n}}+\left\|p^{+}\right\|_{A_{+}^{m-1 ; n-1}}\right) \\
& \left.+\left(\left((\ln M)^{\frac{1}{2}}+1\right) M^{\frac{1}{2}-\frac{r}{2}}+N^{-s}\right)\left\|\boldsymbol{f}^{+}\right\|_{\bar{B}_{+}^{r ; s}}\right] .
\end{aligned}
$$


Proof Let

$$
V_{\mathcal{N}}:=\left\{\boldsymbol{v}_{\mathcal{N}} \in X_{\mathcal{N}}:\left(\nabla \cdot \boldsymbol{v}_{\mathcal{N}}, q\right)_{\mathcal{N}}=0, \forall q \in M_{\mathcal{N}}\right\}
$$

For any $\boldsymbol{u} \in H_{0}^{1}(\Omega)^{2}$, we define

$$
\pi_{\boldsymbol{u}}= \begin{cases}\pi_{\boldsymbol{u}}^{-}=\Pi_{N-1, y}^{1,0} \Pi_{N-1, x}^{1} \boldsymbol{u}, & \boldsymbol{x} \in \Omega^{-}, \\ \pi_{\boldsymbol{u}}^{+}=\Pi_{N-1, y}^{1,0} \hat{\pi}_{M, x}^{1} \boldsymbol{u}, & \boldsymbol{x} \in \Omega^{+},\end{cases}
$$

where $\Pi_{N-1, y}^{1,0}$ and $\Pi_{N-1, x}^{1}$ are the operators $\Pi_{N-1}^{1,0}$ and $\Pi_{N-1}^{1}$ in the $y$ and $x$ directions, respectively. Then, from the properties of the Legendre-Gauss-Lobatto and Laguerre-GaussRadau quadratures, we have $\left(\nabla \pi_{\boldsymbol{u}}, \nabla \boldsymbol{v}_{\mathcal{N}}\right)_{\mathcal{N}}=\left(\nabla \pi_{\boldsymbol{u}}, \nabla \boldsymbol{v}_{\mathcal{N}}\right), \forall \boldsymbol{v}_{\mathcal{N}} \in X_{\mathcal{N}}$. Therefore, for all $\boldsymbol{v}_{\mathcal{N}} \in X_{\mathcal{N}}, \boldsymbol{w}_{\mathcal{N}} \in V_{\mathcal{N}}$, we have

$$
\begin{aligned}
& \left(\nabla \boldsymbol{w}_{\mathcal{N}}, \nabla \boldsymbol{v}_{\mathcal{N}}\right)-\left(\nabla \boldsymbol{w}_{\mathcal{N}}, \nabla \boldsymbol{v}_{\mathcal{N}}\right)_{\mathcal{N}} \\
& \quad=\left(\nabla\left(\boldsymbol{w}_{\mathcal{N}}-\boldsymbol{u}\right), \nabla \boldsymbol{v}_{\mathcal{N}}\right)+\left(\nabla\left(\boldsymbol{u}-\pi_{\boldsymbol{u}}\right), \nabla \boldsymbol{v}_{\mathcal{N}}\right)+\left(\nabla\left(\pi_{\boldsymbol{u}}-\boldsymbol{w}_{\mathcal{N}}\right), \nabla \boldsymbol{v}_{\mathcal{N}}\right)_{\mathcal{N}} \\
& \quad \lesssim\left(\left|\boldsymbol{u}-\boldsymbol{w}_{\mathcal{N}}\right|_{1, \Omega}+\left|\boldsymbol{u}-\pi_{\boldsymbol{u}}\right|_{1, \Omega}\right)\left|\boldsymbol{v}_{\mathcal{N}}\right|_{1, \Omega}+\left|\boldsymbol{w}_{\mathcal{N}}-\pi_{\boldsymbol{u}}\right|_{1, \Omega}\left|\boldsymbol{v}_{\mathcal{N}}\right|_{1, \Omega} \\
& \quad \lesssim\left(\left|\boldsymbol{u}-\boldsymbol{w}_{\mathcal{N}}\right|_{1, \Omega}+\left|\boldsymbol{u}-\pi_{\boldsymbol{u}}\right|_{1, \Omega}\right)\left|\boldsymbol{v}_{\mathcal{N}}\right|_{1, \Omega} .
\end{aligned}
$$

The above result together with Theorem IV.2.5 of [2] leads to

$$
\begin{aligned}
& \left|\boldsymbol{u}-\boldsymbol{u}_{\mathcal{N}}\right|_{1, \Omega} \lesssim \inf _{\boldsymbol{w}_{\mathcal{N}} \in V_{\mathcal{N}}}\left(\left|\boldsymbol{u}-\boldsymbol{w}_{\mathcal{N}}\right|_{1, \Omega}+\sup _{\boldsymbol{v}_{\mathcal{N}} \in X_{\mathcal{N}}} \frac{\left(\nabla \boldsymbol{w}_{\mathcal{N}}, \nabla \boldsymbol{v}_{\mathcal{N}}\right)-\left(\nabla \boldsymbol{w}_{\mathcal{N}}, \nabla \boldsymbol{v}_{\mathcal{N}}\right)_{\mathcal{N}}}{\left|\boldsymbol{v}_{\mathcal{N}}\right|_{1, \Omega}}\right) \\
& +\inf _{q_{\mathcal{N}} \in M_{\mathcal{N}}}\left\|p-q_{\mathcal{N}}\right\|_{0, \Omega}+\sup _{\boldsymbol{v}_{\mathcal{N}} \in X_{\mathcal{N}}} \frac{\left(\boldsymbol{f}, \boldsymbol{v}_{\mathcal{N}}\right)_{\mathcal{N}}-\left(\boldsymbol{f}, \boldsymbol{v}_{\mathcal{N}}\right)}{\left|\boldsymbol{v}_{\mathcal{N}}\right|_{1, \Omega}} \\
& \lesssim \inf _{\boldsymbol{w}_{\mathcal{N}} \in V_{\mathcal{N}}}\left|\boldsymbol{u}-\boldsymbol{w}_{\mathcal{N}}\right|_{1, \Omega}+\left|\boldsymbol{u}-\pi_{\boldsymbol{u}}\right|_{1, \Omega}+\inf _{q_{\mathcal{N}} \in M_{\mathcal{N}}}\left\|p-q_{\mathcal{N}}\right\|_{0, \Omega} \\
& +\sup _{\boldsymbol{v}_{\mathcal{N}} \in X_{\mathcal{N}}} \frac{\left(\boldsymbol{f}, \boldsymbol{v}_{\mathcal{N}}\right)_{\mathcal{N}}-\left(\boldsymbol{f}, \boldsymbol{v}_{\mathcal{N}}\right)}{\left|\boldsymbol{v}_{\mathcal{N}}\right|_{1, \Omega}} ;
\end{aligned}
$$

and

$$
\begin{aligned}
\left\|p-p_{\mathcal{N}}\right\|_{0, \Omega} \lesssim & \frac{1}{\beta_{\mathcal{N}}}\left[\inf _{\boldsymbol{w}_{\mathcal{N}} \in V_{\mathcal{N}}}\left|\boldsymbol{u}-\boldsymbol{w}_{\mathcal{N}}\right|_{1, \Omega}+\left|\boldsymbol{u}-\pi_{\boldsymbol{u}}\right|_{1, \Omega}\right. \\
& \left.+\inf _{q_{\mathcal{N}} \in M_{\mathcal{N}}}\left\|p-q_{\mathcal{N}}\right\|_{0, \Omega}+\sup _{\boldsymbol{v}_{\mathcal{N}} \in X_{\mathcal{N}}} \frac{\left(\boldsymbol{f}, \boldsymbol{v}_{\mathcal{N}}\right)_{\mathcal{N}}-\left(\boldsymbol{f}, \boldsymbol{v}_{\mathcal{N}}\right)}{\left|\boldsymbol{v}_{\mathcal{N}}\right|_{1, \Omega}}\right] .
\end{aligned}
$$

Now we estimate the terms on the right-hand side of the above two inequalities.

Firstly, for all $\boldsymbol{f} \in\left\{\boldsymbol{v}: \boldsymbol{v}^{-} \in H^{\rho}\left(\Omega^{-}\right)^{2}, \boldsymbol{v}^{+} \in \bar{B}^{r ; s}\left(\Omega^{+}\right)^{2}\right\} \cap C^{0}(\bar{\Omega})$, we know from [2]

$$
\left(\boldsymbol{f}^{-}, \boldsymbol{v}_{\mathcal{N}}^{-}\right)_{\mathcal{N},-}-\left(\boldsymbol{f}^{-}, \boldsymbol{v}_{\mathcal{N}}^{-}\right)_{-} \lesssim N^{-\rho}\left\|\boldsymbol{f}^{-}\right\|_{\rho, \Omega^{-}}\left|\boldsymbol{v}_{\mathcal{N}}^{-}\right|_{1, \Omega^{-}}, \quad \forall \boldsymbol{v}_{\mathcal{N}} \in X_{\mathcal{N}}
$$

On the other hand, we have

$$
\begin{aligned}
& \left(\boldsymbol{f}^{+}, \boldsymbol{v}_{\mathcal{N}}^{+}\right)_{\mathcal{N},+}-\left(\boldsymbol{f}^{+}, \boldsymbol{v}_{\mathcal{N}}^{+}\right)_{+} \\
& \quad \lesssim\left(\left\|\boldsymbol{f}^{+}-I_{N, y} \hat{I}_{M, x} \boldsymbol{f}^{+}\right\|_{0, \Omega^{+}}+\left\|\boldsymbol{f}^{+}-\Pi_{N-1, y} \hat{\pi}_{M, x} \boldsymbol{f}^{+}\right\|_{0, \Omega^{+}}\right)\left|\boldsymbol{v}_{\mathcal{N}}^{+}\right|_{1, \Omega^{+}} \\
& \quad \lesssim\left(\left\|\boldsymbol{f}^{+}-I_{N, y} \boldsymbol{f}^{+}\right\|_{0 ; 0}+\left\|\boldsymbol{f}^{+}-\hat{I}_{M, x} \boldsymbol{f}^{+}\right\|_{0 ; 1}+N^{-s}\left\|\boldsymbol{f}^{+}\right\|_{0 ; s}\right.
\end{aligned}
$$




$$
\begin{aligned}
& \left.+M^{\frac{1}{2}-\frac{r}{2}}\left\|\hat{\partial}_{x}^{r} \boldsymbol{f}^{+}\right\|_{\hat{\omega}_{r} ; 0}\right)\left|\boldsymbol{v}_{\mathcal{N}}^{+}\right|_{1, \Omega^{+}} \\
\lesssim & \left(N^{-s}\left\|\boldsymbol{f}^{+}\right\|_{0 ; s}+M^{\frac{1}{2}-\frac{r}{2}}\left(\left\|\hat{\partial}_{x}^{r} \boldsymbol{f}^{+}\right\|_{\hat{\omega}_{r-1} ; 1}+(\ln M)^{\frac{1}{2}}\left\|\hat{\partial}_{x}^{r} \boldsymbol{f}^{+}\right\|_{\hat{\omega}_{r} ; 1}\right)\right. \\
& \left.+N^{-s}\left\|\boldsymbol{f}^{+}\right\|_{0 ; s}+M^{\frac{1}{2}-\frac{r}{2}}\left\|\hat{\partial}_{x}^{r} \boldsymbol{f}^{+}\right\|_{\hat{\omega}_{r} ; 0}\right)\left|\boldsymbol{v}_{\mathcal{N}}^{+}\right|_{1, \Omega^{+}} \\
\lesssim & \left(N^{-s}\left\|\boldsymbol{f}^{+}\right\|_{0 ; s}+M^{\frac{1}{2}-\frac{r}{2}}\left(\left\|\hat{\partial}_{x}^{r} \boldsymbol{f}^{+}\right\|_{\hat{\omega}_{r-1} ; 1}+(\ln M)^{\frac{1}{2}}\left\|\hat{\partial}_{x}^{r} \boldsymbol{f}^{+}\right\|_{\hat{\omega}_{r} ; 1}\right)\right)\left|\boldsymbol{v}_{\mathcal{N}}^{+}\right|_{1, \Omega^{+}} .
\end{aligned}
$$

So we get

$$
\begin{aligned}
& \left(\boldsymbol{f}, \boldsymbol{v}_{\mathcal{N}}\right)_{\mathcal{N}}-\left(\boldsymbol{f}, \boldsymbol{v}_{\mathcal{N}}\right) \\
& \quad=\left[\left(\boldsymbol{f}^{-}, \boldsymbol{v}_{\mathcal{N}}^{-}\right)_{\mathcal{N},-}-\left(\boldsymbol{f}^{-}, \boldsymbol{v}_{\mathcal{N}}^{-}\right)_{-}\right]+\left[\left(\boldsymbol{f}^{+}, \boldsymbol{v}_{\mathcal{N}}^{+}\right)_{\mathcal{N},+}-\left(\boldsymbol{f}^{+}, \boldsymbol{v}_{\mathcal{N}^{+}}^{+}\right)_{+}\right] \\
& \quad \lesssim\left(N^{-\rho}\left\|\boldsymbol{f}^{-}\right\|_{\rho, \Omega^{-}}+\left(\left((\ln M)^{\frac{1}{2}}+1\right) M^{\frac{1}{2}-\frac{r}{2}}+N^{-s}\right)\left\|\boldsymbol{f}^{+}\right\|_{\bar{B}_{+}^{r ; s}}\right)\left|\boldsymbol{v}_{\mathcal{N}}\right|_{1, \Omega}
\end{aligned}
$$

Secondly, by the approximation property of $\Pi_{N, y}^{1,0}$ and $\hat{\pi}_{M, x}^{1}$, we find

$$
\begin{aligned}
\left\|\frac{\partial}{\partial x}\left(\boldsymbol{u}^{+}-\pi_{\boldsymbol{u}}^{+}\right)\right\|_{0, \Omega^{+}} & \leq\left\|\frac{\partial}{\partial x}\left(\boldsymbol{u}-\hat{\pi}_{M, x}^{1} \boldsymbol{u}\right)\right\|_{0, \Omega^{+}}+\left\|\frac{\partial}{\partial x} \hat{\pi}_{M, x}^{1}\left(\boldsymbol{u}-\Pi_{N-1, y}^{1,0} \boldsymbol{u}\right)\right\|_{0, \Omega^{+}} \\
& \lesssim M^{\frac{1}{2}-\frac{m}{2}}\left\|\hat{\partial}_{x}^{m} \boldsymbol{u}\right\|_{\hat{\omega}_{m-1} ; 0, \Omega^{+}}+N^{1-n}\left\|\frac{\partial}{\partial x} \hat{\pi}_{M, x}^{1} \boldsymbol{u}\right\|_{0 ; n-1, \Omega^{+}} \\
& \lesssim M^{\frac{1}{2}-\frac{m}{2}}\left\|\hat{\partial}_{x}^{m} \boldsymbol{u}\right\|_{\hat{\omega}_{m-1} ; 0, \Omega^{+}}+N^{1-n}\left\|\hat{\partial}_{x} \boldsymbol{u}\right\|_{\omega_{0} ; n-1, \Omega^{+}}, \\
\left\|\frac{\partial}{\partial y}\left(\boldsymbol{u}^{+}-\pi_{\boldsymbol{u}}^{+}\right)\right\|_{0, \Omega^{+}} & \leq\left\|\frac{\partial}{\partial y}\left(\boldsymbol{u}-\Pi_{N-1, y}^{1,0} \boldsymbol{u}\right)\right\|_{0, \Omega^{+}}+\left\|\frac{\partial}{\partial y} \Pi_{N-1, y}^{1,0}\left(\boldsymbol{u}-\hat{\pi}_{M, x}^{1} \boldsymbol{u}\right)\right\|_{0, \Omega^{+}} \\
& \lesssim N^{1-n}\|\boldsymbol{u}\|_{0 ; n, \Omega^{+}}+M^{\frac{1}{2}-\frac{m}{2}}\left\|\hat{\partial}_{x}^{m} \partial_{y} \boldsymbol{u}\right\|_{\hat{\omega}_{m-1} ; 0, \Omega^{+}} .
\end{aligned}
$$

From the above two estimates, we get

$$
\left|\boldsymbol{u}^{+}-\pi_{\boldsymbol{u}}^{+}\right|_{1, \Omega^{+}} \lesssim\left(N^{1-n}+M^{\frac{1}{2}-\frac{m}{2}}\right)\|\boldsymbol{u}\|_{Y_{+}^{m, n}} .
$$

Similarly, by the approximation property of $\Pi_{N}^{1}$ and $\Pi_{N}^{1,0}$ in Lemmas 4.5 and 4.6, we find

$$
\left|\boldsymbol{u}^{-}-\pi_{\boldsymbol{u}}^{-}\right|_{1, \Omega^{-}} \lesssim N^{1-\sigma}\|\boldsymbol{u}\|_{\sigma, \Omega^{-}} .
$$

Thus, we obtain

$$
\begin{aligned}
\left|\boldsymbol{u}-\pi_{\boldsymbol{u}}\right|_{1, \Omega} & \lesssim\left|\boldsymbol{u}^{-}-\pi_{\boldsymbol{u}}^{-}\right|_{1, \Omega^{-}}+\left|\boldsymbol{u}^{+}-\pi_{\boldsymbol{u}}^{+}\right|_{1, \Omega^{+}} \\
& \lesssim N^{1-\sigma}\|\boldsymbol{u}\|_{\sigma, \Omega^{-}}+\left(N^{1-n}+M^{\frac{1}{2}-\frac{m}{2}}\right)\|\boldsymbol{u}\|_{Y_{+}^{m, n}}
\end{aligned}
$$

Now, by Lemma IV.1.2 of [2], Theorem 4.1 of [1] and Lemma 4.5, we have

$$
\begin{aligned}
\inf _{\boldsymbol{w}_{\mathcal{N}} \in V_{\mathcal{N}}}\left|\boldsymbol{u}-\boldsymbol{w}_{\mathcal{N}}\right|_{1, \Omega} & \leq\left(1+\frac{1}{\beta_{\mathcal{N}}}\right) \inf _{\boldsymbol{w}_{\mathcal{N}} \in X_{\mathcal{N}}}\left|\boldsymbol{u}-\boldsymbol{w}_{\mathcal{N}}\right|_{1, \Omega} \\
& \lesssim \frac{1}{\beta_{\mathcal{N}}}\left|\boldsymbol{u}^{-}-\Pi_{N-1, y}^{1,0} \Pi_{N-1, x}^{1} \boldsymbol{u}^{-}\right|_{1, \Omega^{-}}+\left|\boldsymbol{u}^{+}-\hat{\pi}_{M, x}^{1,0} \Pi_{N-1, y}^{1,0} \boldsymbol{u}^{+}\right|_{1, \Omega^{+}}
\end{aligned}
$$




$$
\lesssim \beta_{\mathcal{N}}^{-1}\left[N^{1-\sigma}\left\|\boldsymbol{u}^{-}\right\|_{\sigma, \Omega^{-}}+\left(M^{\frac{1}{2}-\frac{m}{2}}+N^{1-n}\right)\left\|\boldsymbol{u}^{+}\right\|_{Y_{+}^{m ; n}}\right]
$$

It now remains to estimate $\inf _{q_{\mathcal{N}} \in M_{\mathcal{N}}}\left\|p-q_{\mathcal{N}}\right\|_{0, \Omega}$. By the approximation results of $\hat{\pi}_{M, x}$ and $\Pi_{N, z}$, we have

$$
\begin{aligned}
\inf _{q_{\mathcal{N} \in M_{\mathcal{N}}}\left\|p-q_{\mathcal{N}}\right\|_{0, \Omega}} & \leq\left\|p^{-}-\Pi_{N-2, y} \Pi_{N-2, x} p^{-}\right\|_{0, \Omega^{-}}+\left\|p^{+}-\Pi_{N-2, y} \hat{\pi}_{M-1, x} p^{+}\right\|_{0, \Omega^{+}} \\
& \lesssim N^{1-\sigma}\left\|p^{-}\right\|_{\sigma-1, \Omega^{-}}+N^{1-n}\left\|p^{+}\right\|_{0 ; n-1, \Omega^{+}}+\left\|p^{+}-\hat{\pi}_{M-1, x} p^{+}\right\|_{0 ; \Omega^{+}} \\
& \lesssim N^{1-\sigma}\left\|p^{-}\right\|_{\sigma-1, \Omega^{-}}+N^{1-n}\left\|p^{+}\right\|_{0 ; n-1, \Omega^{+}}+M^{\frac{1}{2}-\frac{m}{2}}\left\|\hat{\partial}_{x}^{m-1} p^{+}\right\|_{\hat{\omega}_{m-2} ; 0} \\
& \lesssim N^{1-\sigma}\left\|p^{-}\right\|_{\sigma-1, \Omega^{-}}+\left(M^{\frac{1}{2}-\frac{m}{2}}+N^{1-n}\right)\left\|p^{+}\right\|_{A_{+}^{m-1 ; n-1}} .
\end{aligned}
$$

As a direct consequence of the above estimates, we finally obtain

$$
\begin{aligned}
\left\|\boldsymbol{u}-\boldsymbol{u}_{\mathcal{N}}\right\|_{1, \Omega} \lesssim & N^{1-\sigma}\left(\beta_{\mathcal{N}}^{-1}\left\|\boldsymbol{u}^{-}\right\|_{\sigma, \Omega^{-}}+\left\|p^{-}\right\|_{\sigma-1, \Omega^{-}}\right)+N^{-\rho}\left\|\boldsymbol{f}^{-}\right\|_{\rho, \Omega^{-}} \\
& +\left(M^{\frac{1}{2}-\frac{m}{2}}+N^{1-n}\right)\left(\beta_{\mathcal{N}^{-1}}\left\|\boldsymbol{u}^{+}\right\|_{Y_{+}^{m ; n}}+\left\|p^{+}\right\|_{A_{+}^{m-1 ; n-1}}\right) \\
& +\left(\left((\ln M)^{\frac{1}{2}}+1\right) M^{\frac{1}{2}-\frac{r}{2}}+N^{-s}\right)\left\|\boldsymbol{f}^{+}\right\|_{\bar{B}_{+}^{r ; s}} \\
\left\|p-p_{\mathcal{N}}\right\|_{0, \Omega} \lesssim & \beta_{\mathcal{N}}^{-1}\left[N^{1-\sigma}\left(\beta_{\mathcal{N}}^{-1}\left\|\boldsymbol{u}^{-}\right\|_{\sigma, \Omega^{-}}+\left\|p^{-}\right\|_{n-1, \Omega^{-}}\right)+N^{-\rho}\left\|\boldsymbol{f}^{-}\right\|_{\rho, \Omega^{-}}\right. \\
& +\left(M^{\frac{1}{2}-\frac{m}{2}}+N^{1-n}\right)\left(\beta_{\mathcal{N}}^{-1}\left\|\boldsymbol{u}^{+}\right\|_{Y_{+}^{m ; n}}+\left\|p^{+}\right\|_{A_{+}^{m-1 ; n-1}}\right) \\
& \left.+\left(\left((\ln M)^{\frac{1}{2}}+1\right) M^{\frac{1}{2}-\frac{r}{2}}+N^{-s}\right)\left\|\boldsymbol{f}^{+}\right\|_{\bar{B}_{+}^{r ; s}}\right]
\end{aligned}
$$

\section{Numerical Computations}

We present in this section several numerical tests to illustrate the theoretical results established in previous sections and to demonstrate how the coupled Legendre-Laguerre spectral element method can be used to simulate realistic flow problems in unbounded domains.

\subsection{Numerical Computation of the inf-sup Constant}

We first compute the inf-sup constant $\beta_{\mathcal{N}}$ described in Sect. 3. To this end, we consider the case that $a=2$ and the domain is decomposed into two elements only.

Inserting the expansions of $\boldsymbol{u}_{\mathcal{N}}$ and $p_{\mathcal{N}}$ into (2.4), the resulting set of algebraic equations can be written in a matrix form,

$$
\begin{aligned}
\boldsymbol{A}_{\mathcal{N}} \underline{\boldsymbol{u}}_{\mathcal{N}}+\boldsymbol{D}_{\mathcal{N}} \underline{p}_{\mathcal{N}}=\boldsymbol{B}_{\mathcal{N}} \underline{\boldsymbol{f}}_{\mathcal{N}}, \\
\boldsymbol{D}_{\mathcal{N}}^{T} \underline{\boldsymbol{u}}_{\mathcal{N}}=0,
\end{aligned}
$$

where $\underline{f}_{\mathcal{N}}$ is a vector representation of the $f$ at the LGL and LGR nodes. The matrices $\boldsymbol{A}_{\mathcal{N}}, \boldsymbol{D}_{\mathcal{N}}$ and $\boldsymbol{B}_{\mathcal{N}}$ are block-diagonal matrices with 2 blocks each. The blocks of $\boldsymbol{A}_{\mathcal{N}}$ are the discrete Laplace operators, and those of $\boldsymbol{D}_{\mathcal{N}}$ are associated to the different components 
of the discrete gradient operators, while blocks of $\boldsymbol{B}_{\mathcal{N}}$ are the mass matrices with respect to each component of $\boldsymbol{f}$.

Eliminating $\underline{\boldsymbol{u}}_{\mathcal{N}}$ from (5.7)-(5.8), we obtain

$$
\underbrace{\boldsymbol{D}_{\mathcal{N}}^{T} \boldsymbol{A}_{\mathcal{N}}^{-1} \boldsymbol{D}_{\mathcal{N}}}_{\boldsymbol{S}_{\mathcal{N}}} \underline{p}_{\mathcal{N}}=\boldsymbol{D}_{\mathcal{N}^{T}}^{T} \boldsymbol{A}_{\mathcal{N}}^{-1} \boldsymbol{B}_{\mathcal{N}} \underline{\boldsymbol{f}}_{\mathcal{N}} .
$$

The matrix $\boldsymbol{S}_{\mathcal{N}}:=\boldsymbol{D}_{\mathcal{N}}^{T} \boldsymbol{A}_{\mathcal{N}}^{-1} \boldsymbol{D}_{\mathcal{N}}$ is usually referred as the Uzawa matrix. A typical procedure for solving (5.7)-(5.8) is to first solve $\underline{p}_{\mathcal{N}}$ from (5.9), and then solve $\underline{\boldsymbol{u}}_{\mathcal{N}}$ from the Poisson equation (5.7) with known $\underline{p}_{\mathcal{N}}$.

The Uzawa matrix is of dimension $(M+N-1) \times(N-1)$, full, symmetric and positive definite. A usual procedure to solve (5.9) is to use a preconditioned conjugate gradient method with the mass matrix $\widetilde{\boldsymbol{B}}_{\mathcal{N}}$ as a preconditioner $[2,5,7,15]$. In this procedure each outer iteration requires the inversion of two Laplace operators $\left(\boldsymbol{A}_{\mathcal{N}}\right.$ matrix $)$ which can be carried out by the fast diagonalization method (see [13]). Hence, the efficiency of the method is dictated by the condition number $\kappa_{\mathcal{N}}$ of $\widetilde{\boldsymbol{B}}_{\mathcal{N}}^{-1} \boldsymbol{S}_{N}$. A well known fact is that the condition number $\kappa_{\mathcal{N}}$ is linked to the inf-sup constant via the relation $\kappa_{\mathcal{N}}=\frac{1}{\beta_{\mathcal{N}}^{2}}$ [15]. As a result, the inf-sup constant $\beta_{\mathcal{N}}$ affects the efficiency of the iteration method to solving (5.9).

The first computational investigation is concerned with the lower bound on the inf-sup constant derived in Sect. 3. In the left of Fig. 2, we plot the variations of $\beta_{\mathcal{N}}$ versus $M$ in $\log$-log scale for several $N$. We observe that $\beta_{\mathcal{N}}$ is independent of $N$ while it decays as $\frac{1}{M}$. In the right of Fig. 2, we plot the variations of $\beta_{\mathcal{N}}$ versus $N$ for several $M$. We observe that $\beta_{\mathcal{N}}$ remains to be constants as we vary $N$ with $M$ fixed. These results seem to indicate that $\beta_{\mathcal{N}}=O\left(\frac{1}{M}\right)$, which is the same as in the coupled Laguerre-Legendre approximation in [1]. However, we were not able to improve our estimate in Sect. 3 to match this numerical observation. There are possibly two scenarios for this discrepancy: (i) the numerical results show in Fig. 2 have not reached the asymptotic range; and (ii) the lower bound for the infsup constant in Sect. 3 is not optimal. In any case, this is an issue of academic interest only since in practice we always use $M \geq \sqrt{N}$ so $\beta_{\mathcal{N}} \sim \min \left(N^{-1 / 2}, M^{-1}\right) \sim M^{-1}$.
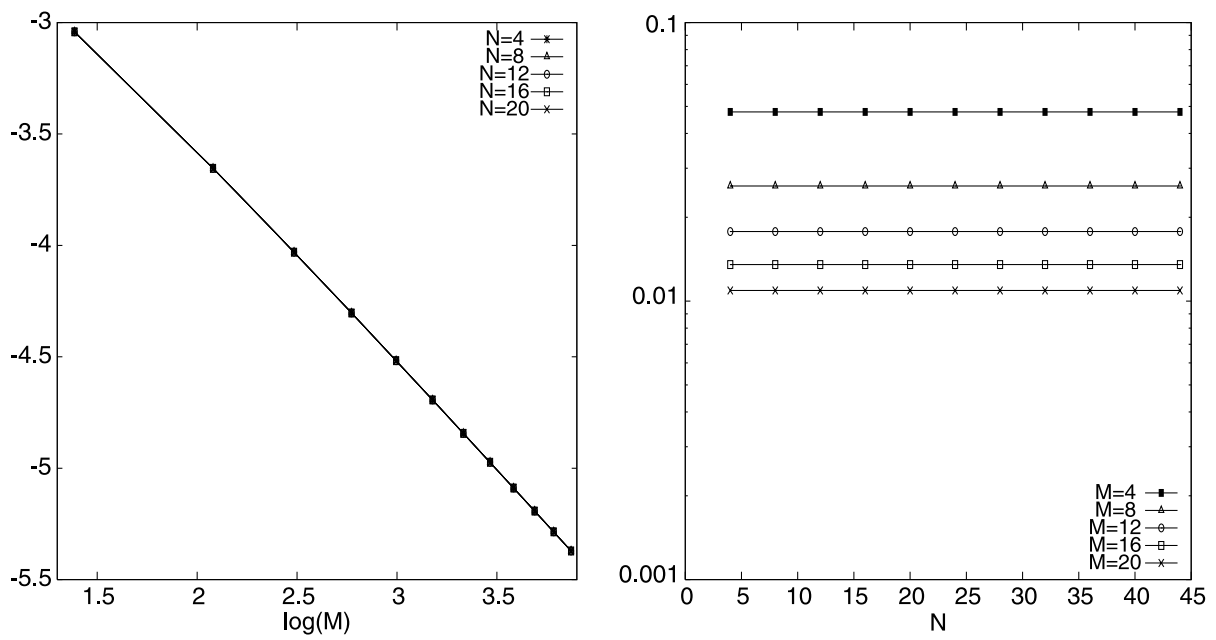

Fig. 2 Left: inf-sup constant $\beta_{\mathcal{N}}$ vs. $M$; right: inf-sup constant $\beta_{\mathcal{N}}$ vs. $N$ 


\subsection{Numerical Tests for the Convergence Rate}

The next numerical test is carried out to check the convergence behavior of the coupled Legendre-Laguerre spectral element method in case of a more general domain decomposition. To this end, we divide $\Omega^{-}$and $\Omega^{+}$into several non-overlapping macro-elements.

To simplify the presentation, we assume that all elements are rectilinear. We partition $\Omega^{-}$ into $K^{-}$non-overlapping elements:

$$
\bar{\Omega}^{-}=\bigcup_{k=1}^{K^{-}} \bar{\Omega}_{k}^{-}, \quad \Omega_{k}^{-} \cap \Omega_{l}^{-}=\emptyset, \quad \text { for } k \neq l,
$$

i.e., we partition $\Lambda_{a}=(0, a)$ in $x$ direction into $K_{x}$ parts:

$$
\bar{\Lambda}_{a}=\bigcup_{k=1}^{K_{x}} \bar{\Lambda}_{a}^{k}, \quad \Lambda_{a}^{k} \cap \Lambda_{a}^{l}=\emptyset, \quad \text { for } k \neq l,
$$

while partition $\Lambda=(-1,1)$ in $y$ direction into $K_{y}$ parts:

$$
\bar{\Lambda}=\bigcup_{k=1}^{K_{y}} \bar{\Lambda}^{k}, \quad \Lambda^{k} \cap \Lambda^{l}=\emptyset, \quad \text { for } k \neq l .
$$

Obviously, we have $K^{-}=K_{x} \times K_{y}$. At the same time, we divide $\Omega^{+}$into $K^{+}=K_{y}$ nonoverlapping parts, i.e., we divide $y$ direction of $\Omega^{+}$as in $\Omega^{-}$. The finial domain configuration is illustrated below:

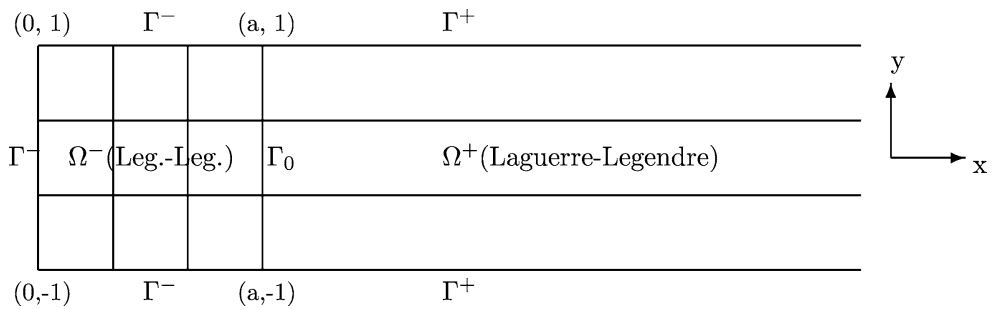

We consider the Stokes problem with the following analytical solution

$$
\boldsymbol{u}=\left(\begin{array}{c}
\sin (x) \cos (y) e^{-x} \\
(\sin (x)-\cos (x)) \sin (y) e^{-x}
\end{array}\right), \quad p=\cos (x) \cos (y) e^{-x}
$$

in the computation domain $\Omega$ that is the union of the following two sub-domains:

$$
\Omega^{-}=\{\boldsymbol{x}: 0<x<2,-1<y<1\}, \quad \Omega^{+}=\{\boldsymbol{x}: 2<x<\infty,-1<y<1\} .
$$

Then we further decompose $\Omega^{-}$into 16 equal macro-elements, and $\Omega^{+}$into 4 equal macroelements. In Fig. 3, we plot, in a semi-logarithmic scale, the $H^{1}$-velocity and the $L^{2}$-pressure errors in $\Omega^{-}$as a function of $N$ with $M$ fixed (figure on the left) and in $\Omega^{+}$as a function of $M$ with $N$ fixed (figure on the right). We observe that the errors converge exponentially which is a typical behavior for spectral methods with analytical solutions. 

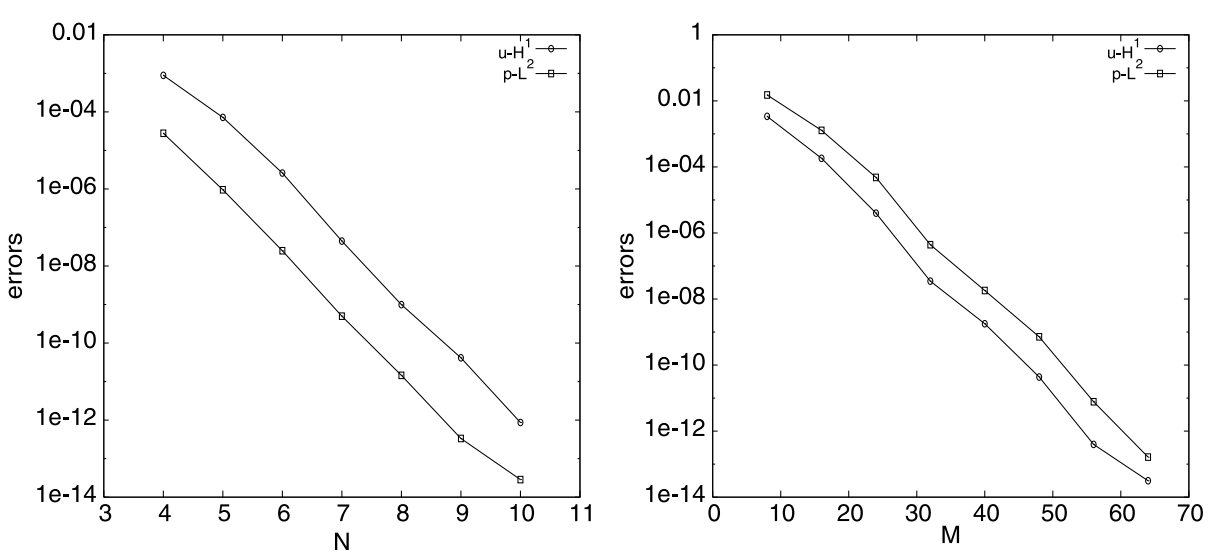

Fig. 3 The velocity and pressure errors as a function of $N$ and $M$ : left, errors in $\Omega^{-}$; right, errors in $\Omega^{+}$

\subsection{Applications to Flow Simulations}

We now consider simulations of fluid flows passing a cylinder. To understand what and how the computations will be done, we review below the time discretization algorithm to be used here.

We consider the flow of an incompressible fluid around a circular cylinder placed in a semi-infinite channel. The flow is governed by the following Navier-Stokes equations:

$$
\begin{cases}\frac{\partial u}{\partial t}-\frac{1}{R e} \Delta \boldsymbol{u}+(\boldsymbol{u} \cdot \nabla) \boldsymbol{u}+\nabla p=\boldsymbol{f} & \text { in } \Omega, \\ \nabla \cdot \boldsymbol{u}=0 & \text { in } \Omega,\end{cases}
$$

where $\boldsymbol{u}=\left(u_{1}, u_{2}\right), p$ are respectively the non-dimensional velocity and pressure, $\boldsymbol{f}$ is the forcing term. The characteristic scales for the velocity and pressure are respectively the freestream velocity $U$ and $\rho_{0} U^{2}$, where $\rho_{0}$ is the density. $R e:=\frac{U D}{v}$ is the Reynolds number, with $D$ the cylinder diameter and $v$ the kinematic viscosity. In our simulations, the free-stream velocity is set to the constant $(1,0)$. This means that during the calculations the velocity remains this constant in the infinity downstream. In order to be able to use our method, we define a new vector function $\boldsymbol{w}=\left(w_{1}, w_{2}\right):=\left(u_{1}-1, u_{2}\right)$ so that $\lim _{x \rightarrow \infty} \boldsymbol{w}=0$, which is required by the Laguerre approximation. Then $(5.10)$ becomes

$$
\begin{cases}\frac{\partial \boldsymbol{w}}{\partial t}-\frac{1}{R e} \Delta \boldsymbol{w}+\left(w_{1}+1, w_{2}\right) \cdot \nabla \boldsymbol{w}+\nabla p=\boldsymbol{f} & \text { in } \Omega, \\ \nabla \cdot \boldsymbol{w}=0 & \text { in } \Omega .\end{cases}
$$

Usually, the nonlinear terms are treated explicitly either via a Adams-Bashforth scheme or a characteristics scheme based on the operator-integration-factor (OIF) splitting method [14]. In most applications, the latter has shown advantage over the former thanks to the fact that the OIF method allows for time-step sizes exceeding standard CFL limited time-step sizes. For this reason, we use a semi-Lagrangian method (see, e.g., [19]), which is based on the OIF splitting technique, to treat the time derivative and the convective terms. This temporal discretization results in a saddle point problem coupling the velocity and the pressure, which is decoupled later via an additional splitting step. Such an approach was analyzed and applied to various computations in the papers of Perot [16], Couzy et al. [6], and Lin et al. [12]. The approach has a common foundation with traditional projection approaches which 
Fig. 4 Partition of domain and a typical mesh
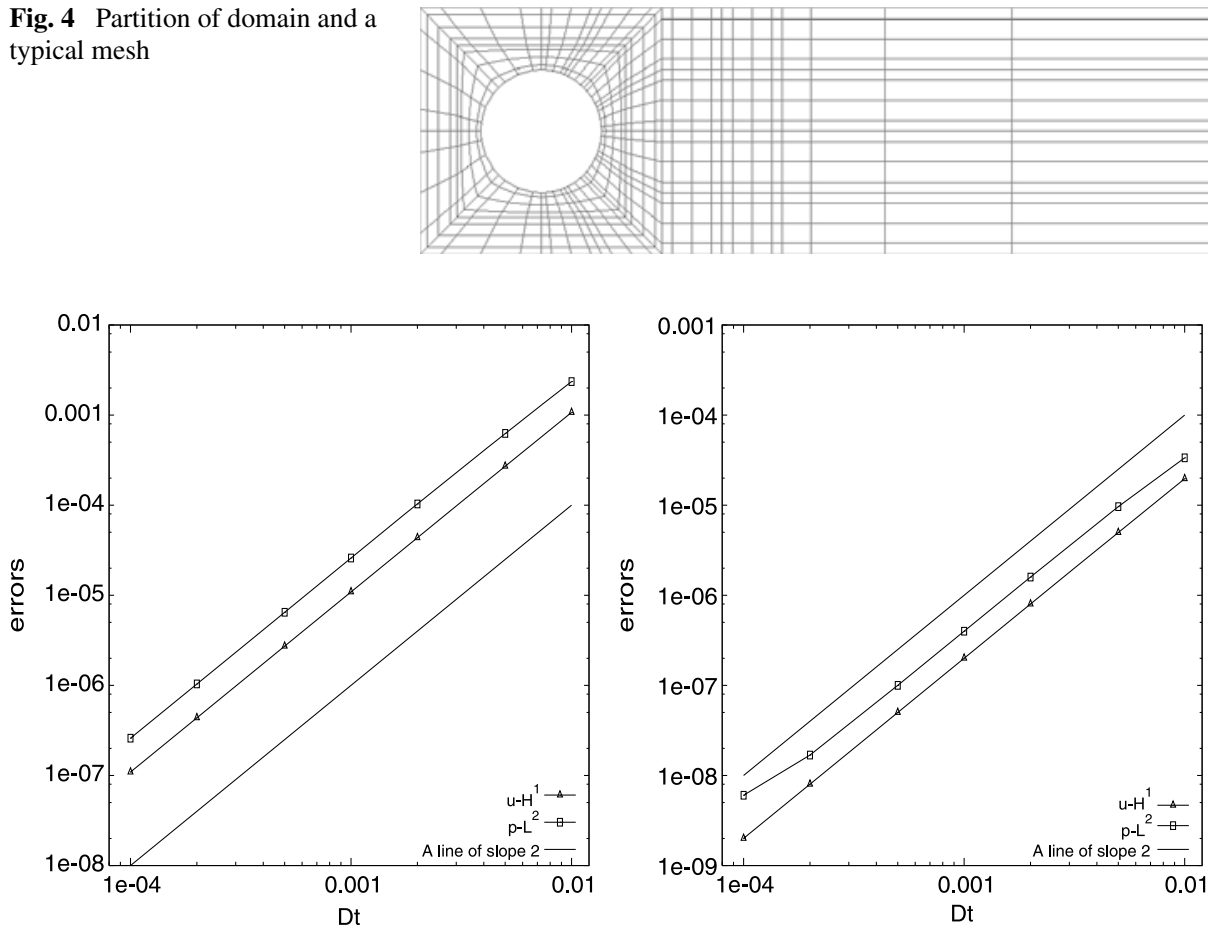

Fig. 5 The velocity and pressure errors as a function of $\Delta t$ : left, errors in $\Omega^{-}$; right, errors in $\Omega^{+}$

lead to a Poisson equation for the pressure (cf. [9]). More precisely, we rewrite (5.11) under the following form:

$$
\begin{cases}\frac{D w}{D t}-\frac{1}{R e} \Delta \boldsymbol{w}+\nabla p=\boldsymbol{f} & \text { in } \Omega, \\ \nabla \cdot \boldsymbol{w}=0 & \text { in } \Omega,\end{cases}
$$

where $\frac{D}{D t}$ denotes the material derivative taken along a path moving with velocity $\left(w_{1}+1, w_{2}\right)$. Then we use the second order backward differentiation scheme to discretize (5.12)

$$
\begin{cases}\frac{3 \boldsymbol{w}^{n+1}-4 \tilde{\boldsymbol{w}}^{n}+\tilde{\boldsymbol{w}}^{n-1}}{2 \Delta t}-\frac{1}{R e} \Delta \boldsymbol{w}^{n+1}+\nabla p^{n+1}=2 \boldsymbol{f}^{n}-\boldsymbol{f}^{n-1} & \text { in } \Omega, \\ \nabla \cdot \boldsymbol{w}^{n+1}=0 & \text { in } \Omega,\end{cases}
$$

where $\Delta t$ is the time step, $\tilde{\boldsymbol{w}}^{n}$ and $\tilde{\boldsymbol{w}}^{n-1}$ are the transports at different instants of the previous solutions on the characteristics. This time semi-discretization leads to a generalized Stokes equation for $\left(\boldsymbol{w}^{n+1}, p^{n+1}\right)$ in each time step, which is further discretized in space by using the coupled Legendre-Laguerre spectral element method described in the preceding sections.

We shall first test this approach with an analytical solution given by

$$
\boldsymbol{u}=\left(\begin{array}{c}
\cos (t) \sin (x) \cos (y) e^{-x} \\
\cos (t)(\sin (x)-\cos (x)) \sin (y) e^{-x}
\end{array}\right), \quad p=\cos (t) \cos (x) \cos (y) e^{-x}
$$

We take the computational domain to be $\{x: 0<x<\infty,-1<y<1\} \backslash \Omega_{0}$ with $\Omega_{0}=$ $\left\{(x, y):(x-1)^{2}+(y-1)^{2} \leq 0.5^{2}\right\}$. The computational domain $\Omega$ is first decomposed into 

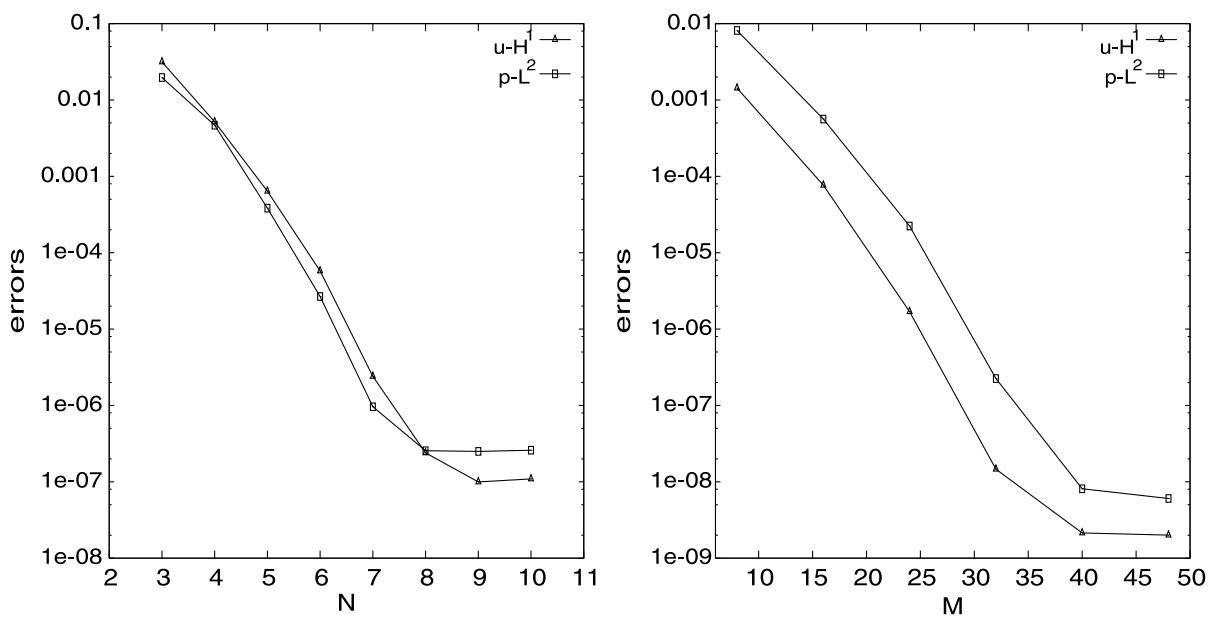

Fig. 6 The velocity and pressure errors as a function of $N$ and $M$ : left, errors in $\Omega^{-}$; right, errors in $\Omega^{+}$

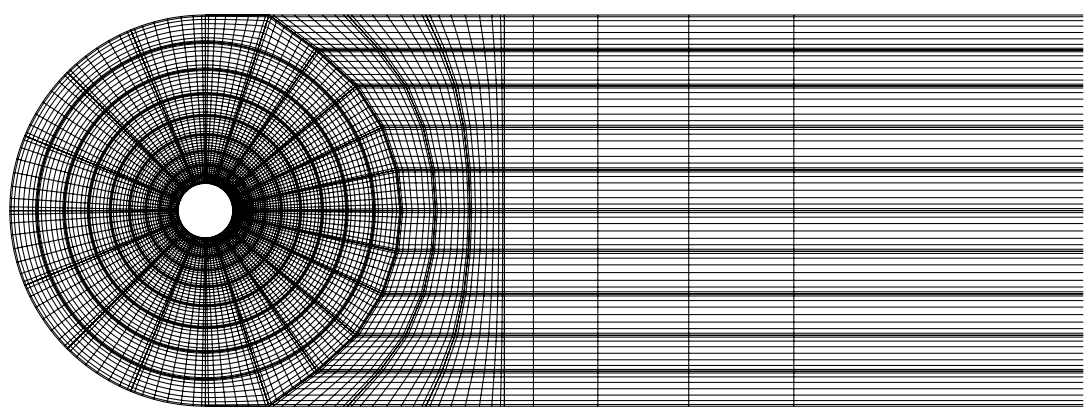

Fig. 7 Legendre-Gauss-Lobatto/Laguerre-Gauss-Radau spectral element mesh for the unbounded domain

Fig. 8 Angle-velocity of the cylinder rotation

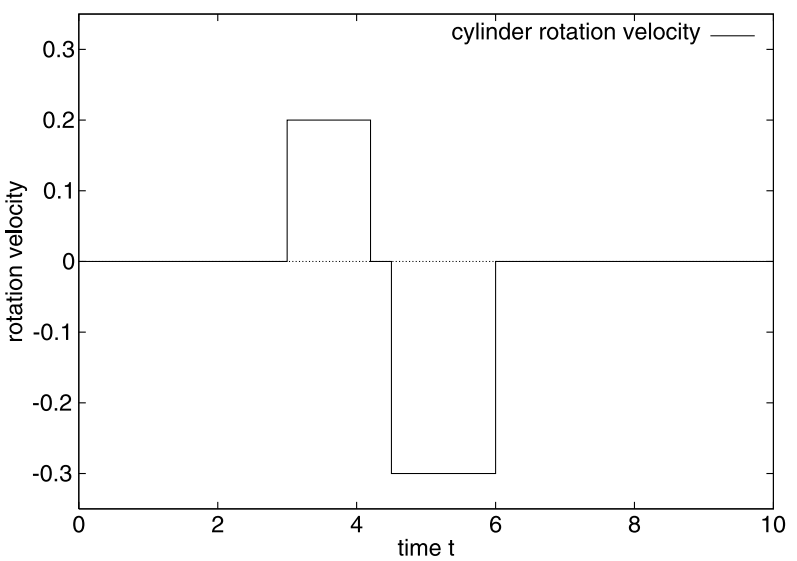


Fig. 9 Standard

Legendre-Gauss-Lobatto spectral element mesh for the bounded domain

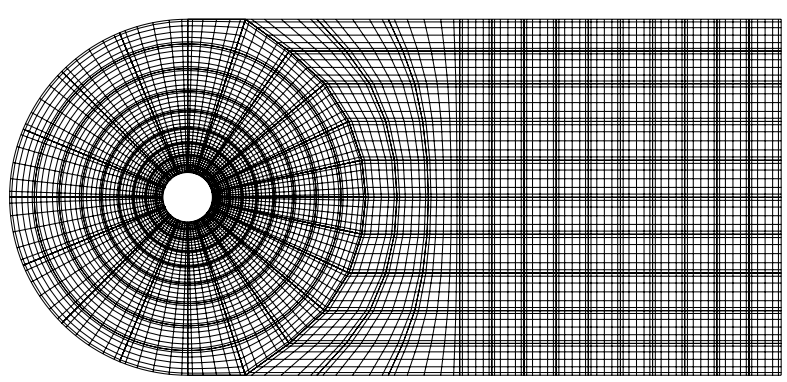

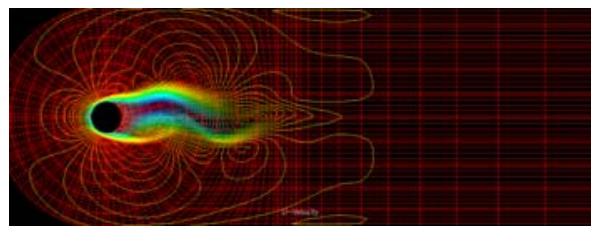

(a) Horizontal velocity, Leg.-Lag.

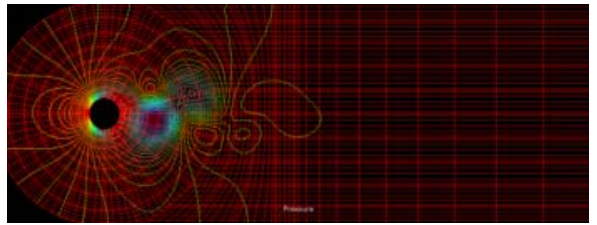

(c) Pressure, Leg.-Lag.

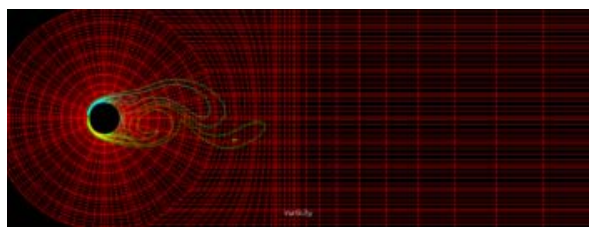

(e) Vorticity, Leg.-Lag.

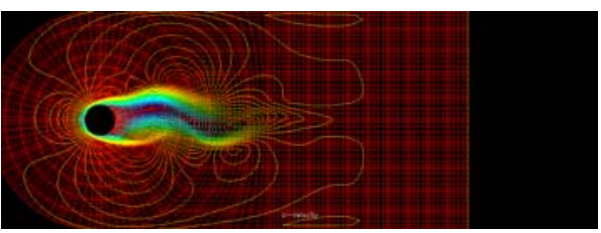

(b) Horizontal velocity, Legendre

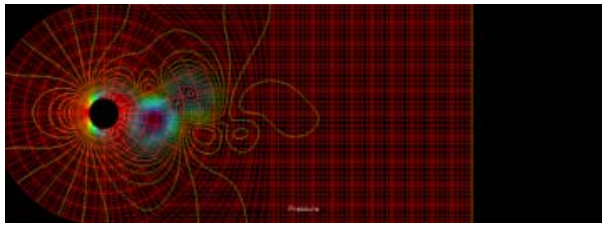

(d) Pressure, Legendre

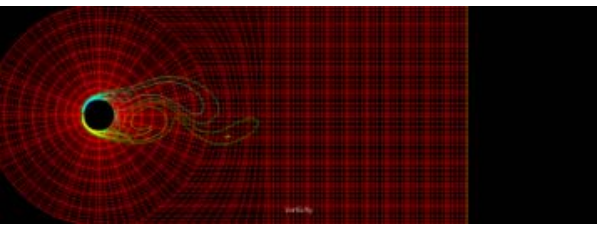

(f) Vorticity, Legendre

Fig. 10 Isolines of the horizontal velocity (a), (b), pressure $(\mathbf{c}),(\mathbf{d})$, and vorticity (e), (f) at $t=10$. Left: Legendre-Laguerre coupled method; right: Legendre method

two sub-domains:

$$
\Omega^{-}=\{x: 0<x<2,-1<y<1\} \backslash \Omega_{0}, \quad \Omega^{+}=\{x: 2<x<\infty,-1<y<1\} .
$$

Then we further partition $\Omega^{-}$into a number of macro-elements, and $\Omega^{+}$into several equal macro-elements. In this case deformed elements have to be used to partition the domain. A typical mesh for this problem is shown in Fig. 4.

We show in Fig. 5 the errors at $t=1$ in $\Omega^{-}$and $\Omega^{+}$as a function of the time step $\Delta t$ with $N=10, M=48$ fixed, second order accuracy in time is observed. 


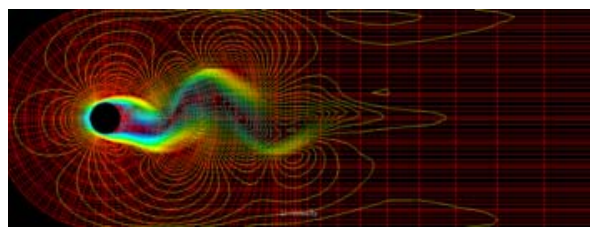

(a) Horizontal velocity, Leg.-Lag.

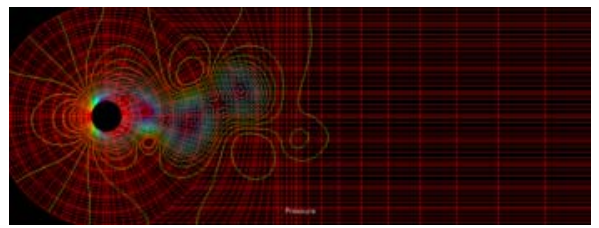

(c) Pressure, Leg.-Lag.

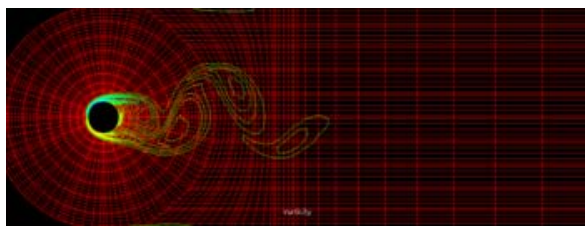

(e) Vorticity, Leg.-Lag.

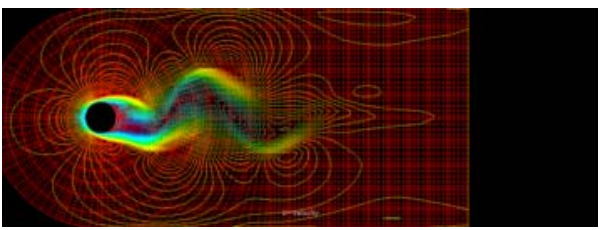

(b) Horizontal velocity, Legendre

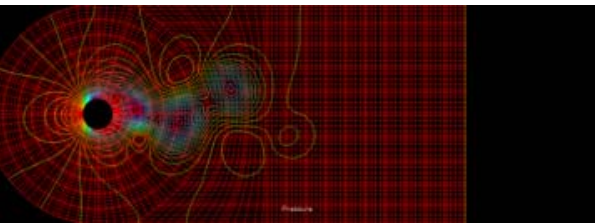

(d) Pressure, Legendre

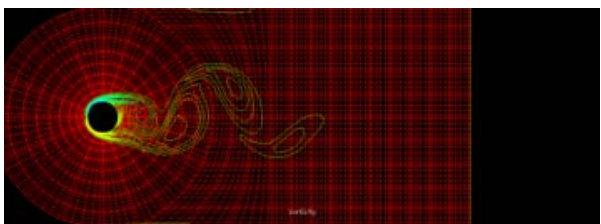

(f) Vorticity, Legendre

Fig. 11 Same as in Fig. 10, but $t=12$

We now consider the flow passing a circular cylinder. The computational domain and the spectral element mesh are illustrated in Fig. 7 , with $\Omega^{+}=(6.15, \infty) \times(-3.6,3.6)$. A cylinder obstacle of diameter $D=1$ is located at the origin. There are 210 Legendre-Legendre macro-elements and 10 Laguerre-Legendre macro-elements extending to the infinity.

All the computations have been carried out for a Reynolds number of 200 by using our coupled method with the Legendre polynomial degree $N=6$, Laguerre polynomial degree $M=32$, and the time step $\Delta t=0.02$. In order to accelerate vortex formation, the cylinder is artificially perturbed by performing impulsively started rotations with a clockwise anglevelocity 0.2 between $t=3.0$ and 4.2, and a counterclockwise angle-velocity 0.3 between $t=4.5$ and 6.0 (cf. Fig. 8).

The simulation results obtained in the unbounded domain are compared to the ones computed by a standard Legendre spectral element method in a bounded domain using a nonreflective artificial boundary condition [20]. A standard Legendre spectral element mesh for the bounded domain is shown in Fig. 9. The bounded domain is partitioned into 310 Legendre-Legendre macro-elements.

In Fig. 10, we plot the isolines of the horizontal velocity, pressure, and vorticity at $t=10$, obtained respectively by using the coupled Legendre-Laguerre spectral element method and the standard Legendre spectral element method. One observes a very good agreement between the two families of plots. The isolines at $t=12$ are plotted in Fig. 11. It is seen that the results produced by the two methods remains indistinguishable. The computation is con- 


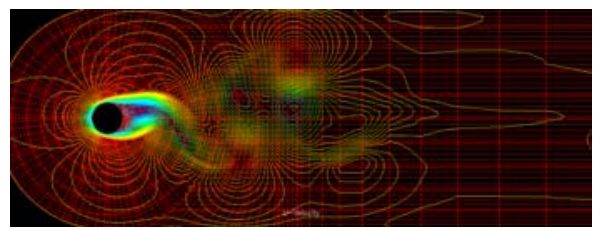

(a) Horizontal velocity, Leg.-Lag.

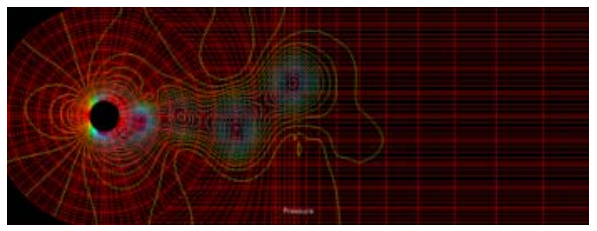

(c) Pressure, Leg.-Lag.

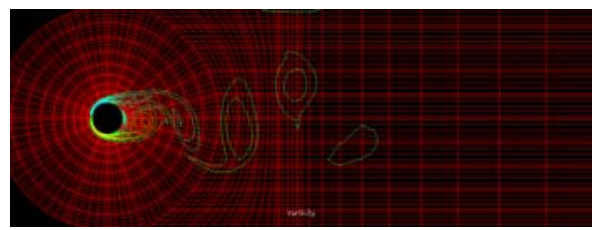

(e) Vorticity, Leg.-Lag.

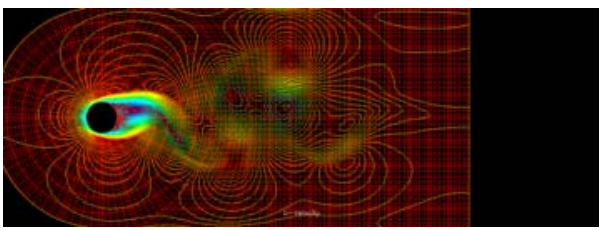

(b) Horizontal velocity, Legendre

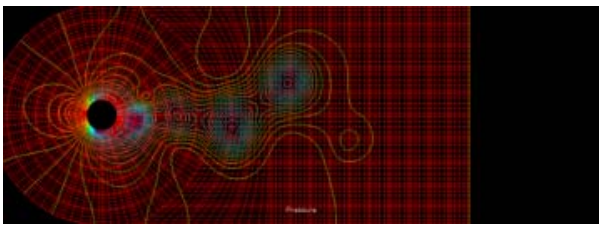

(d) Pressure, Legendre

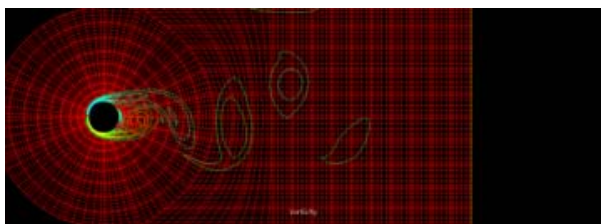

(f) Vorticity, Legendre

Fig. 12 Same as in Fig. 11, but $t=14$

tinued until the vortices reach the downstream domain. We present the results at $t=14$ in Fig. 12 where some vortices appear in the downstream.

We observe here some slight differences between the two approaches at downstream. This is not surprising since the Legendre spectral element method uses, at the artificial outflow boundary, a non-reflecting boundary condition which is no longer valid when some vortices reach the artificial boundary.

In Fig. 6, we present the errors at $t=1$ with respect to $N$ in $\Omega^{-}$with $M=48$ (figure on the left), and with respect to $M$ in $\Omega^{+}$with $N=10$ (figure on the right). One observes that the errors decay exponentially until the time discretization errors dominate. These results demonstrate that the Legendre-Laguerre coupled spectral element method for unbounded domains possesses same convergence features as those of the conventional Legendre spectral element methods for bounded domains.

\section{Concluding Remarks}

We have presented a coupled Legendre-Laguerre spectral element method for the Stokes and Navier-Stokes equations in a semi-infinite domain. A detailed stability and error analysis has been carried out for the Stokes problem in a semi-infinite channel. Namely, we established the well-posedness of this method by deriving an explicit lower bound for the inf-sup con- 
stant, and derived error estimates by using the inf-sup estimate and the Laguerre/Legendre approximation properties.

We have also implemented the new method to simulate the flow passing a circular cylinder, and compared the results with those obtained by using the standard Legendre spectral element method which uses a non-reflecting boundary condition at an artificial boundary. The new method enjoys the following advantages: (i) no artificial boundary is needed as the method works directly in the infinite domain, eliminating the ad-hoc process of choosing an artificial boundary and boundary conditions; (ii) the interface between the Legendre spectral-element region and the Legendre-Laguerre region can be placed very close to the obstacle, leading to potentially significant saving in the number of unknowns and CPU.

\section{References}

1. Azaiez, M., Shen, J., Xu, C., Zhuang, Q.: A Laguerre-Legendre spectral method for the Stokes problem in a semi-infinite channel. SIAM J. Numer. Anal. 47(1), 271-292 (2008)

2. Bernardi, C., Maday, Y.: Approximations Spectrales de Problèmes aux Limites Elliptiques. Springer, Berlin (1992)

3. Boyd, J.P.: Rational Chebyshev spectral methods for unbounded solutions on an infinite interval using polynomial-growth special basis functions. Comput. Math. Appl. 41, 1293-1315 (2001)

4. Brezzi, F.: On the existence, uniqueness and approximation of saddle-point problems arising from Lagrangian multipliers. Rev. Fr. Autom. Informat. Rech. Opér. Sér. Rouge 8(R-2), 129-151 (1974)

5. Canuto, C., Hussaini, M.Y., Quarteroni, A., Zang, T.A.: Spectral Methods in Fluid Dynamics. Springer, Berlin (1987)

6. Couzy, W., Deville, M.O.: A fast schur complement method for the spectral element discretization of the incompressible Navier-Stokes equations. J. Comput. Phys. 116, 135-142 (1995)

7. Deville, M.O., Fischer, P.F., Mund, E.H.: High-Order Methods for Incompressible Fluid Flow. Cambridge Monographs on Applied and Computational Mathematics, vol. 9. Cambridge University Press, Cambridge (2002)

8. Funaro, D.: Polynomial Approximations of Differential Equations. Springer, Berlin (1992)

9. Guermond, J.L., Minev, P., Shen, J.: An overview of projection methods for incompressible flows. Comput. Methods Appl. Mech. Eng. 195, 6011-6045 (2006)

10. Guo, B.-Y., Wang, L.-L.: Jacobi approximations in non-uniformly Jacobi-weighted Sobolev spaces. J. Approx. Theory 128(1), 1-41 (2004)

11. Guo, B.-Y., Wang, L.-L., Wang, Z.-Q.: Generalized Laguerre interpolation and pseudospectral method for unbounded domains. SIAM J. Numer. Anal. 43(6), 2567-2589 (2006)

12. Lin, Y., Xu, C.: A fractional step method for the time dependent incompressible Navier-Stokes/Euler coupled equations. Acta Aerodyn. Sin. 21(3), 368-376 (2003)

13. Lynch, R.E., Rice, J.R., Thomas, D.H.: Direct solution of partial differential equations by tensor product methods. Numer. Math. 6, 185-199 (1964)

14. Maday, Y., Patera, A.T., Rønquist, E.M.: An operator integration-factor splitting method for timedependent problems: application to incompressible fluid flow. J. Sci. Comput. 5, 263-292 (1990)

15. Maday, Y., Meiron, D., Patera, A.T., Rønquist, E.M.: Analysis of iterative methods for the steady and unsteady Stokes problem: application to spectral element discretizations. SIAM J. Sci. Comput. 14(2), 310-337 (1993)

16. Perot, J.B.: An analysis of the fractional step method. J. Comput. Phys. 108, 51-58 (1993)

17. Shen, J.: Stable and efficient spectral methods in unbounded domains using Laguerre functions. SIAM J. Numer. Anal. 38(4), 1113-1133 (2000)

18. Shen, J., Wang, L.-L.: Some recent advances on spectral methods for unbounded domains. Commun. Comput. Phys. 5, 195-241 (2009)

19. Xu, C., Pasquetti, R.: On the efficiency of semi-implicit and semi-Lagrangian spectral methods for the calculation of incompressible flows. Int. J. Numer. Methods Fluids 35, 319-340 (2001)

20. Xu, C., Lin, Y.: A numerical comparison of outflow boundary conditions for spectral element simulations of incompressible flows. Commun. Comput. Phys. 2, 477-500 (2007) 